Journal of Sound \& Vibration; doi:10.1016/j.jsv.2011.10.015

\title{
Mechanical Impedances Distributed at the Fingers and Palm of the Human Hand in Three Orthogonal Directions
}

\author{
Ren G. Dong*, Daniel E. Welcome*, Xueyan S. Xu*, Christopher Warren*, \\ Thomas W. McDowell*, John Z. Wu*, and Subhash Rakheja** \\ *Engineering \& Control Technology Branch \\ National Institute for Occupational Safety and Health \\ Morgantown, West Virginia 26505, USA \\ **Department of Mechanical Engineering \\ Concordia University \\ Montreal, Quebec, Canada
}

\begin{abstract}
The objective of this study is to investigate the basic characteristics of the three axis mechanical impedances distributed at the fingers and palm of the hand subjected to vibrations along the three orthogonal directions $\left(x_{h}, y_{h}\right.$, and $\left.z_{h}\right)$. Seven subjects participated in the experiment on a novel threedimensional (3-D) hand-arm vibration test system equipped with a 3-D instrumented handle. The total impedance of the entire hand-arm system was obtained by performing a sum of the distributed impedances. Two major resonances were observed in the impedance data in each direction. For the hand forces (30 N grip and $50 \mathrm{~N}$ push) and body postures applied in this study, the first resonance was in the range of 20 to $40 \mathrm{~Hz}$, and it was primarily observed in the impedance at the palm. The second resonance was generally observed in the impedance at the fingers, while the resonance frequency varied greatly with the subject and vibration direction, ranging from 100 to $200 \mathrm{~Hz}$ in the $x_{h}$ direction, 60 to $120 \mathrm{~Hz}$ in the $y_{h}$ direction, and 160 to $300 \mathrm{~Hz}$ in the $z_{h}$ direction. The impedance at the palm was greater than that at the fingers below a certain frequency in the range of 50 to $100 \mathrm{~Hz}$, depending on the vibration direction. At higher frequencies, however, the impedance magnitude at the fingers either approached or exceeded that at the palm. The impedance in the $z_{h}$ direction was generally higher than those in the other directions, but it became comparable with that in the $x_{h}$ direction at frequencies above $250 \mathrm{~Hz}$, while the impedance in the $y_{h}$ direction was the lowest. The frequency dependencies of the vibration power absorptions for the entire hand-arm system in the three directions were different, but their basic trends were similar to that of the frequency weighting defined in the current ISO standard. The implications of the results are discussed.
\end{abstract}


Key words: mechanical impedance of the hand-arm system, biodynamic response of the hand-arm system, finger impedance, hand-arm vibration, hand-transmitted vibration.

\section{Introduction}

Prolong, intensive exposure to hand-transmitted vibration may cause hand-arm vibration syndrome [1]. The study of biodynamic responses of the hand-arm system to vibration has been considered vital for understanding mechanisms of vibration-induced disorders [2]. When the hands are coupled to a vibrating tool or machine, the dynamic behavior of the tool or machine can be affected by the biodynamic properties of the hand-arm system. Hence, knowledge of the biodynamic response is one of the essential foundations for the further development of more effective methods for assessing vibration exposure risks and for developing better tools and vibration-reducing devices $[3,4]$.

Because vibration-induced stresses, strains, and their time rate of change are among the essential mechanical stimuli that could directly cause the vibration-induced psychophysical, physiological, and pathological responses of the hand-arm system [2,5], the major objectives of vibration biodynamics are to quantify the stimuli for determining the vibration exposure dose and to help establish relationships between the exposure dose and the vibration-induced psychophysical responses and health effects. While a reliable method for directly measuring these detailed responses has not yet been developed, their quantifications have mostly relied upon the development and analysis of the hand-arm system models [6,7], which remains a formidable research task. As part of the process, the human hand-arm responses to vibration have been widely investigated in terms of driving-point biodynamic response, which can be directly measured from the contact dynamic force and the motion at the contact interface [8]. The driving-point biodynamic response yields essential information on the responses in the hand substructures in the vicinity of the driving point, and is considered vital for the development of biomechanical models. 
While the driving-point biodynamic responses such as the biodynamic force at the interface and the vibration power absorption of the hand-arm system can be used as measures of vibration exposure, the response functions such as apparent mass, mechanical impedance and dynamic stiffness of the system represent the overall biodynamic properties of the system. The response function in terms of the mechanical impedance has been most frequently studied. On the basis of the synthesis of the reported mechanical impedance data by Gurram et al. [9], the International Organization for Standardization (ISO) has set forth the ranges of mechanical impedance and the mechanical equivalent models of the hand-arm system under vibration applied along the individual $x_{h^{-}}, y_{h^{-}}$and $z_{h^{-}}$axis [4]. Since this standard was published, a few studies have shown considerable limitations of the recommended models for applications in hand-arm simulator designs $[10,11]$, while the recommended impedance data vary over wide ranges $[9,12]$. Apart from these, some concerns exist on the applicability and/or validity of reported biodynamic response functions [11,12]. A few studies have also investigated the effects of some of the influencing factors such as hand and arm posture, applied hand forces, vibration spectra, and tool operating orientations on the biodynamic response functions [13-16]. However, the vast majority of the recently reported studies have focused on the responses along the $z_{h}$-axis. There is lack of data in the other two orthogonal directions $\left(x_{h}, y_{h}\right)$ for the revision of the current standard. Therefore, more reliable experimental measurements of the responses and modeling studies are desirable to help improve the standard. 
The majority of the driving-point biodynamic response data have been derived assuming vibration excitation at a single-point at the hand-handle interface $[8,9,13-16]$. The reported responses can thus be considered to represent the overall response of the entire hand-arm system and have been widely applied to derive values for the total vibration power absorbed into the hand-arm system. While the total biodynamic response could be associated with the overall subjective sensation or discomfort [17], it does not yield information on the vibration responses of specific hand substructures. According to SaintVenant's principle [18], the single-point approach may be acceptable to analyze the biodynamic responses in the arms and shoulders but it is not acceptable when the responses in the vicinity of contact substructures, especially the fingers, are of concern. The single-point approach also makes it impossible to correctly predict the vibration transmissibility of anti-vibration gloves at the palm or at the fingers. A recent study by Dong et al. [19] showed that application of the mechanical impedance distributed at the palm, as opposed to the overall hand-arm impedance recommended in ISO-13753 [20], would yield a more reliable prediction of the glove vibration transmissibility compared with that measured at the palm using the method defined ISO 10819 (1996) [21].

Several investigators hypothesized that the vibration power absorption (VPA) may be a significant etiological factor with regards to potential vibration injuries. The overall impedance response of the hand-arm system has been widely used to determine the total VPA of the entire hand-arm system as a measure of the vibration exposure [22-24]. While this hypothesis is consistent with the concept of vibration biomechanics and the vibration power density (VPA per unit volume of tissue $=$ strain rate $\times$ 
stress) may be a good measure of the vibration mechanical stimuli, it is questionable whether the total VPA approach is generally applicable, especially in regards to vibration-induced finger injuries and disorders [25]. Although none of the reported studies have actually established a reliable relationship between the total VPA and any finger disorder, some researchers further hypothesized that the measurement of the energy absorbed in the hand and arm may be a better and more objective method for risk assessment than the measurement of the ISO frequency-weighted acceleration [23,26,27]. Recent studies, however, contradicted this hypothesis and demonstrated that the total VPA in the $z_{h}$ direction could be very similar to the frequency-weighted acceleration [17]. Further studies are required to clarify whether the frequency dependencies of the total VPAs in the other directions would also be similar to the frequency weighting defined in ISO 5349-1 (2001) [27].

As an approach to overcome the deficiency of the single-point approach and to help resolve the abovementioned issues, a two-point approach has been proposed [28]. This approach separates the handhandle interface into two distinct driving-points: one at the palm and one at the fingers. A few two-point models have also been proposed, which demonstrated more realistic simulations of the hand-arm system [29]. One of these models has been used to predict the VPA distributed across the major substructures of the hand-arm system along the forearm direction [30]. It has been further developed to analyze the basic vibration isolation mechanism of anti-vibration gloves [19]. However, only preliminary results on the distributed responses in the other vibration directions have been reported [31,32]. 
Powered hand tools are known to generate and transmit multi-axes vibrations. Due to its elastic and viscous properties, the human hand-arm system could respond to such multi-axes vibrations in a highly complex manner along all the translational and rotational axes. Largely because of lack of reliable multiaxis hand-arm test equipment, the mechanical impedance of the hand-arm system has been almost exclusively measured on a single-axis vibration test system. This approach ignores the dynamic coupling between the biodynamic responses along different axes. It is also very difficult to apply a push force in the measurement of the impedance in the directions vertical to the forearm axis on a single-axis test system. A novel three-dimensional (3-D) hand-arm test system has been developed and it has made it possible to overcome these deficiencies [33].

On the basis of the above review, the primary objective of this study was formulated to examine fundamental characteristics of the driving-point mechanical impedances distributed at the fingers and palm of the hand subjected to random vibrations in three orthogonal directions. The measurements are performed on the 3-D hand-arm vibration test system. The results are used to identify the basic characteristics of the biodynamic responses and to understand their associations with the dynamic properties of the hand-arm system. The experimental data are also used to derive the frequency dependencies of the vibration power absorptions of the entire hand-arm system in the three directions and to explore their relationships with the ISO frequency-weighted acceleration. The implications of the results are also discussed. 


\section{Method}

\subsection{Subjects}

Seven healthy male subjects participated in the experiment of the study. Table 1 summarizes the essential attributes of the subjects together with their hand lengths and breadths. The experimental protocol was reviewed and approved by the human subject review board of the National Institute for Occupational Safety and Health.

\subsection{Experimental Setup and Evaluation Method}

The instrumentation setup and the subject posture used in this study for measuring the driving-point mechanical impedances distributed at the fingers and palm of the hand-arm system are illustrated in Fig. 1. The 3-D vibration test system (MB Dynamics, 3-D Hand-Arm Vibration Test System) was employed to generate the required 3-D vibration spectra. Fig. 2 shows a pictorial view of the mechanical configuration of the test system, together with the human hand-arm coupling the handle. As shown in this figure, the $z_{h}$ direction is along the forearm, $y_{h}$ direction is along the centerline of the instrumented handle in the vertical direction and $x_{h}$ direction is in the horizontal plane normal to $y_{h}-z_{h}$ plane.

A special instrumented handle was designed and constructed to fit the 3-D test system for measuring the 3-D biodynamic responses distributed at the palm and the fingers. As illustrated in Fig. 3, the handle is composed of a measuring cap, a handle base, and the attachments. A tri-axial accelerometer (ENDEVCO 65-100) mounted on the measuring cap served as the primary feedback for synthesizing the desired vibration spectra along the three axes. The dynamic force developed at the driving-point was measured using a pair of 3-D force sensors (Kistler 9017B and 9018B) installed within the handle. The grip force exerted on the handle along the forearm direction was also measured from these force sensors. Specifically, it was represented using the quasi-static component $(<5 \mathrm{~Hz})$ of the total force signal in the $\mathrm{Z}_{\mathrm{h}}$-axis. A force plate (Kistler 9286AA) was used to measure the push force applied to the handle. The measured grip and push forces in their time histories were averaged at every 0.2 second interval and displayed on dial gages to the subject, as shown in Fig. 1, which helped the subject to maintain the desired forces. The instrumented handle design permitted the measurements of biodynamic force developed either at the palm-handle or the fingers-handle interface, depending upon the orientation of the measuring cap as seen in Fig. 3. This allowed for measurements of biodynamic responses distributed at the palm and fingers of the hand, which were acquired in a sequential manner. The biodynamic responses distributed at the palm of the seven subjects were initially measured. Subsequently, the instrumented handle was rotated $180^{\circ}$ to realize fingers contact with the measuring cap for measurement of response distributed at the fingers.

In this study, each subject was instructed to maintain the grip and push forces as $30 \pm 5 \mathrm{~N}$ and $50 \pm 8 \mathrm{~N}$, respectively. Each subject was also instructed to grip the handle with his right hand with the forearm parallel to the floor and aligned with the $z_{h}$-axis, The elbow angled between $90^{\circ}$ and $120^{\circ}$, and shoulder 
abducted between $0^{\circ}$ and $30^{\circ}$, similar to the posture used for the reference values in ISO 10068 (1998) [4] and that recommended in the standardized glove test (ISO 10819, 1996) [21].

The 3-D vibration test system used in this study is limited from its allowable vibration displacement and its fundamental resonant frequency $(630 \mathrm{~Hz})$. Considering that the dominant vibration frequencies of the vast majority of the tools are in the range of 25 to $300 \mathrm{~Hz}$ [1,34], and that the important resonances of hand and fingers are also in the same frequency range [14-16,28-30], the frequency range used in this study was from 16 to $500 \mathrm{~Hz}$. A multi-axis vibration controller was programmed to generate a broadband random vibration in this frequency range. The overall root-mean-square value of the acceleration in each direction was $19.6 \mathrm{~m} / \mathrm{s}^{2}$. While the average coherence for tools used at workplaces remains unknown, it was taken as 0.9 in this study for reliable control of the 3-D excitations without interruption during each trial. This was also based on the considerations that the dominant multi-axes vibrations of a tool are likely to be correlated because they are transmitted from the same vibration sources, and that the variation of the coherence is unlikely to change the basic characteristics of the biodynamic response function. The three-axis force signals, together with the acceleration signals, were acquired in a multi-channel signal analyzer (B\&K Type 3032A I/O Module) while the subject gripped the handle with the prescribed hand forces. The measured signals were analyzed to evaluate the apparent mass of the human hand-arm at the palm and fingers interfaces using the cross-spectrum function available in the B\&K Pulse software (Version 11.0) of the analyzer. The results were expressed in the frequency domain, corresponding to the center frequencies of the one third-octave bands from 16 to 500 $\mathrm{Hz}$.

The directly-measured total apparent mass $\left(M_{T}=\right.$ total dynamic force divided by acceleration $)$ also included the tare mass $\left(M_{C}\right)$ of the measuring cap and portions of the sensor masses [28]. Therefore, the tare mass must be cancelled from the measured total apparent mass in order to derive the apparent mass $\left(M_{A}\right)$ along each direction [28] as follows:

$$
M_{A i}(j \omega)=\frac{\alpha \cdot \operatorname{Mean}\left[\operatorname{Mag}\left(M_{C i}\right)\right]}{M_{C i}(j \omega)} \cdot\left[M_{T i}(j \omega)-M_{C i}(j \omega)\right] ; \quad i=x_{h}, y_{h}, z_{h},
$$

where $\alpha$ is a calibration factor, $\omega$ is the frequency in $\mathrm{rad} / \mathrm{s}, j=\sqrt{-1}, \operatorname{Mean}\left[\operatorname{Mag}\left(M_{C i}\right)\right]$ is the average magnitude of the tare mass $\left(M_{C i}\right)$ measured without the hand coupling along direction $i$ in the frequency range of concern (16 to $500 \mathrm{~Hz}$ ). $M_{A i}$ is the tare mass-corrected apparent mass of the hand, distributed at the palm or the fingers, along direction $i$.

The validity of the measurement system and the mass cancellation method was examined through measurements performed on the handle with a small rigid mass of $31 \mathrm{~g}$ that was firmly attached using elastic bands. The measured total mass was corrected using Eq. (1). The resulting effective mass along each axis revealed peak deviations of less than $5 \%$ from the applied mass $(31 \mathrm{~g})$ in the entire frequency 
range of concern, while the peak phase error was less than $5^{\circ}$, which suggests the measurement system is acceptable.

The mechanical impedance $\left(Z_{i}\right)$ in each direction was derived from the apparent mass using the following relation:

$$
Z_{i}(j \omega)=M_{A i}(j \omega) \cdot j \omega
$$

\subsection{Experimental Procedures}

The measurement of the 3-D biodynamic responses was performed in a well-controlled laboratory environment (room temperature $=22^{\circ}$, relative humidity $=50 \%$ ). Each subject was advised to stand on the force plate adjusted to an appropriate height to assume the desired hand-arm posture and alignment, and to grip and push on the vibrating handle. The acquisition of force and acceleration data was initiated when the hand forces approached their desired values. Each measurement was repeated twice, while the data during each trial was recorded over three durations of $20 \mathrm{~s}$ each. The apparent mass in each direction was linearly averaged during each measurement period. The data acquired during the three durations were further linearly averaged to represent the responses corresponding to each trial.

\subsection{Hand Impedance and Frequency Dependence of Vibration Power Absorption}

It has been shown that the summation of the impedance distributed at the fingers $\left(Z_{F}\right)$ and at the palm $\left(Z_{P}\right)$ yields the driving-point biodynamic response of the entire hand-arm system $\left(Z_{H}\right)$ [28]. The measured distributed responses were thus applied to derive the total response of the system along each axis of vibration from:

$$
Z_{H i}=Z_{F i}+Z_{P i} ; i=x_{h}, y_{h}, z_{h} .
$$

The frequency dependence $\left(W_{\mathrm{VPA}}\right)$ of the vibration power absorption (VPA) along each axis was subsequently derived from the real part of the mechanical impedance as [17]:

$$
W_{\mathrm{VPA} i}(\omega)=W_{\mathrm{ISO}}\left(\omega_{\mathrm{REF}}\right) \frac{\sqrt{\operatorname{Re}\left[Z_{H i}(\omega)\right]}}{\omega} / \frac{\sqrt{\operatorname{Re}\left[Z_{H i}\left(\omega_{\mathrm{REF}}\right)\right]}}{\omega_{\mathrm{REF}}},
$$

where $\omega_{\mathrm{REF}}$ is the frequency of the reference impedance and $W_{\mathrm{ISO}}$ is frequency weighting defined in ISO 5349-1 (2001) [27].

\subsection{Statistical Analysis}

To analyze the effects of vibration frequency and direction on each of the finger, palm, and total impedances, a two-factor repeated-measures mixed-model analysis-of-variance (ANOVA) for the function was performed using a general linear model. In the model, the first fixed factor reflected 
differences in the three orthogonal directions $\left(x_{h}, y_{\mathrm{h}}\right.$, and $\left.z_{h}\right)$, and the second fixed factor reflected the responses at the center frequencies of the 16 one-third octave bands in the 16 to $500 \mathrm{~Hz}$ range. Test subject was included in the statistical model as a random factor.

In similar fashion, the differences among hand substructures and frequencies were examined for each of the impedances in each orthogonal direction using two-factor mixed-model ANOVAs. In the model, the first factor reflected the impedance distribution (palm, finger, and hand), while the second factor reflected the responses at the center frequencies of the 16 one-third octave bands. Again, test subject was included in each statistical model as a random factor.

To find the significances of the direction and distribution at each center frequency, a stratified (one-way) ANOVA was conducted for each frequency. Pursuant to the results of the ANOVAs, pair-wise comparisons were also performed. For all statistical analyses, effects were considered significant when the resultant $p$-value was less than 0.05 .

\section{Results}

Figs. 4 to 6 illustrate the driving-point impedance magnitude and phase responses of the seven subjects along the $x_{h^{-}}, y_{h^{-}}$, and $z_{h}$-axis, respectively. They show the impedance responses measured at the palm and fingers together with total hand-arm system responses. The results show considerable variations in the magnitude and phase responses across the subjects irrespective of the measurement location (palm, fingers and the entire hand-arm system) and the axis of measurement. The statistical results suggest significant effects of exposure direction, exposure frequency, and their interactions, on the impedance magnitude and phase measurements for each hand substructure (fingers, palm, and entire hand) $(F \geq 3$. $04 p<0$. 001). The effects of the impedance distribution (substructure), the exposure frequency, and their interactions are also significant for all the impedance components $(F \geq 2.00 p \leq 0.04)$, except for the frequency $\times$ distribution interaction in the $\mathrm{z}_{\mathrm{h}}$-axis phase response $(F=0.91 \quad p=0.599)$.

Despite the considerable inter-subject variability, the responses measured along each axis consistently exhibit two magnitude peaks in the frequency range considered. The frequencies corresponding to these peaks can be approximately considered as dominant resonance frequencies of the hand-arm system [28,29]. The first magnitude peak is observed in approximately 20 to $40 \mathrm{~Hz}$ frequency range, which varied considerably across the subjects, but it did not vary greatly with vibration direction for the individual subjects. This resonance was primarily reflected in the response at the palm in all three measurement directions and was also evident from the response at the fingers in the $\mathrm{z}_{\mathrm{h}}$ direction, as shown in Fig. 6. The second resonant peak was clearly evident in the response at the fingers in each direction, although it could also be identified in the palm impedance responses in the $x_{h^{-}}$and $z_{h^{-}}$ directions. The corresponding peak frequency varied greatly across the subjects and with vibration direction; it varied from approximately 100 to $200 \mathrm{~Hz}$ in the $\mathrm{x}_{\mathrm{h}^{-}}$-direction, from 60 to $120 \mathrm{~Hz}$ in the $\mathrm{y}_{\mathrm{h}^{-}}$ direction, and from 160 to $300 \mathrm{~Hz}$ in the $z_{h}$-direction. 
Fig. 7 depicts the mean magnitude and phase distributed impedance responses for the fingers, palm, and for their summation (hand) in each of the three orthogonal directions. The results shown in Fig. 7 and those attained from pair-wise comparisons indicate that the magnitudes of the palm impedances in the $x_{h}$ - and $y_{h}$-directions at less than $50 \mathrm{~Hz}$ were greater than those of the fingers impedances at frequencies below $50 \mathrm{~Hz}(p<0.05)$. This suggests that the impedance response of the total hand-arm system primarily arises from that measured at the palm. The palm impedance in the $z_{h}$-direction at frequencies below $100 \mathrm{~Hz}$ obviously played a more dominant role in the total impedance. However, the finger impedance magnitudes are either comparable or greater than the palm impedance magnitudes at higher frequencies (Fig. 7).

The phase angles of the finger impedances are greater than those of the palm impedances at frequencies below $100 \mathrm{~Hz}$ in the $x_{h^{-}}$and $y_{h}$-directions and below $300 \mathrm{~Hz}$ in the $z_{h}$-direction. The $x_{h^{-}}$and $y_{h^{-}}$-axis finger impedance phase angles were greater than $90^{\circ}$ at frequencies less than $50 \mathrm{~Hz}$ for some of the subjects (Figs. 4 and 5). This is also evident from the mean $y_{h}$-axis fingers impedance phase response presented in Fig. 7. The ANOVA results also indicate that the finger impedance phase response was generally different from the palm impedance phase at frequencies less than $160 \mathrm{~Hz}$ in each direction $(p<$ 0.05). However, the phase differences in each direction became small or insignificantly different at higher frequencies.

Fig. 8 shows the effect of vibration direction on the finger, palm, and total hand impedance magnitude and phase responses. The results from the pair-wise comparisons indicate that the palm impedance magnitude in the $z_{h}$-direction was considerably larger than those for the other two directions at frequencies below $100 \mathrm{~Hz}(p<0.001)$. At frequencies below $25 \mathrm{~Hz}$, the palm impedance magnitudes in the $y_{h}$ direction was not significantly different from that in the $x_{h}$ direction $(p>0.05)$. At higher frequencies, the $y_{h}$-axis palm impedance magnitude approaches its peak near $30 \mathrm{~Hz}$. Furthermore, the magnitude of $y_{h}$-axis palm impedances is considerably lower than those in the other directions at frequencies above $100 \mathrm{~Hz}$. On the other hand, the magnitudes $x_{h^{-}}$and $z_{h^{-}}$axis palm impedances are comparable at frequencies above $100 \mathrm{~Hz}$. Furthermore, the palm impedance phase responses approach similar values at higher frequencies in all the three directions $(p>0.05)$.

Similar trends are also observed in the impedance responses distributed at the fingers in all three directions. Specifically, the fingers impedance magnitude in the $z_{h}$-direction is higher than those in the other two directions at frequencies below $40 \mathrm{~Hz}(p<0.05)$. In this frequency range, the fingers impedance magnitudes in the $x_{h}$ - and $y_{h}$-directions are not significantly different $(p>0.10)$. While the finger impedance magnitude in the $y_{h}$-direction is the lowest at frequencies above $125 \mathrm{~Hz}$, the finger impedance magnitudes in the $z_{h}$ and $x_{h}$ directions are not significantly different at frequencies higher than $250 \mathrm{~Hz}(p \geq 0$. 10). At such frequencies, the finger impedance phase responses in all the three directions were not significantly different $(p \geq 0$. 05). 
Because the impedance of the hand is primarily distributed at the palm at the low and middle frequencies, the characteristics of the hand impedance in this frequency range are similar to those of the palm impedance, as also shown in Fig. 8. Because the $z_{h}$ and $x_{h}$-axes impedances at the fingers and palm at frequencies higher than $250 \mathrm{~Hz}$ are very similar, the hand impedances in these two directions at the high frequencies are also very similar.

The frequency dependencies of the vibration power absorptions of the entire hand-arm system in the three directions, derived from Eq.(5), are illustrated in Fig. 9, together with the frequency weighting defined in ISO 5349-1 (2001) [27]. Similar to the ISO weighting, the frequency dependencies of the VPA generally decreased with the increase in the frequency, while the rate of decay varied with the vibration direction. The frequency dependence of the $z_{h}$-axis VPA was quite consistent with that of the ISO weighting function in nearly the entire frequency range considered in this study. The $x_{h}$-axis VPA, however, was comparable with the ISO weighting only up to $63 \mathrm{~Hz}$, while its magnitude was larger at higher frequencies. In the $y_{h}$ direction, the frequency dependence of the VPA suggested a higher weighting in the entire frequency range above $20 \mathrm{~Hz}$, while the general trend is comparable with that of the ISO weighting.

\section{Discussion}

This study investigated the translational mechanical impedances distributed at the fingers and the palm of the hand subjected to the vibrations in three orthogonal directions. The basic characteristics of the distributed impedances were identified. They can be used to understand the some important dynamic features of the hand-arm system subjected to a multi-axis vibration exposure.

\subsection{Comparisons of the measured and reported data}

Fig. 10 compares the fingers and palm impedances measured on the 3-D test system in this study with those reported under $z_{h}$-axis vibration alone for similar hand coupling forces, vibration magnitude, and hand-arm posture [17]. As anticipated, the mean values at the majority of the frequencies in the onethird octave bands are statistically different $(p<0$. 05). The differences could in part be attributed to differences in the subjects used in the two studies. As shown in Figs. 4-6, the impedances among the subjects could be substantially different $(>50 \%)$ at some frequencies, although the variations of the subject anthropometry (Table 1) might not be very large. The cross-axis couplings among the responses to 3-D vibrations could be another source of the differences [31]. The differences in part could also arise from the measurement errors that could vary by instrumented handle and the vibration distribution on the handle [35]. However, the comparisons shown in Fig. 10 also indicate that the basic trends of the impedances measured on the 1-D and 3-D test systems are similar. The major resonant frequencies at both the fingers and the palm are similar. The distribution features of the impedances at the fingers and the palm of the hand are also similar. 
While such comparisons for the palm and finger impedances could not be performed for the other directions due to lack of the $x_{h^{-}}$and $y_{h^{-}}$-axis data, Fig. 11 shows comparisons of the mean total impedance magnitude and phase responses measured in this study with the mean and ranges of idealized impedance recommended in ISO-10068 (1998) [4]. The impedance magnitudes in the $x_{h^{-}}$and

$y_{h}$-directions, measured in this study, are surprisingly similar to the reference values recommended in the standard under single-axis vibration. The fundamental trends in the phase responses are also quite similar. The basic trends in $\mathrm{z}_{\mathrm{h}}$-axis impedance are also similar but the magnitudes of the fundamental resonance are largely different. This is because this resonance was not reflected in some of the data used in the synthesis of the recommended values [9,11]. The magnitude and frequency of the resonance observed in this study are similar to those observed in many other studies [9,12,14-16].

The above comparisons suggest that the biodynamic responses to single-axis vibration could be different from those to multi-axis vibrations but the differences are unlikely to change the basic trends and characteristics of the responses. As the first degree of approximation, it seems acceptable to use the single-axis data to represent the responses to multi-axis vibrations. This supports the practice adopted in ISO 10068 (1998) [4,9].

\subsection{Effects of Vibration Direction}

The results of this study confirm that the hand-arm impedance varies with the vibration direction. As shown in Fig. 8, the $z_{h}$-axis impedance magnitude distributed at the palm is considerably larger than those in the other directions at frequencies below $80 \mathrm{~Hz}$. This is most likely caused by the dominant translational responses of the palm-dorsal hand-wrist-forearm subsystem under handle vibration along the forearm direction. The handle vibration also causes lateral or vertical response of the subsystem, similar to the forced 3-D vibrations at the end point of a cantilever beam. Because the moments of inertias of this subsystem in the $x_{h}$ and $y_{h}$ directions are similar, the responses at the palm at low frequencies $(<25 \mathrm{~Hz})$ should be similar, as observed in Fig. 8. With the increase in the frequency, the transmitted vibrations tend to become localized within the hand [36-38], and the effective mass in each direction is no longer dependent on the mass of the wrist and the forearm. In such a case, the impedance magnitudes in the $x_{h}$ and $z_{h}$ directions should become independent of the vibration direction, similar to the response of a ring surrounding the handle. This explains why the impedance magnitudes in the $x_{h}$ and $z_{h}$ directions are similar at higher frequencies, as also seen in Fig. 8. Besides the effective mass and damping, the $y_{h}$ response depends on the shear stiffness of the palmar contact skin. Because the shear stiffness must be less than the compression stiffness in a grip action, the impedance magnitude in the $y_{h}$ direction would be expected to be lower than those in the other directions at higher frequencies, which is also evident in Fig. 8.

\subsection{Distribution of the Driving-point Mechanical Impedance}

As shown in Fig. 10, the distribution characteristics of the impedance in the $z_{h}$-axis in the coupled response are very similar to those observed in the impedance measured with a single $z_{h}$-axis excitation 
[15,28]. Similar trends were also observed in the $x_{h^{-}}$and $y_{h}$-axis responses, as shown in Fig. 7. These observations confirm that the palm impedance in each direction largely depends on the overall response of the palm-dorsal hand-wrist-arm subsystem at lower and middle frequencies. At higher frequencies, however, the distributed responses and resonances primarily depend on the local structure or tissue responses. Because only the soft tissues close to the palm contact area are effectively involved in the response and the palm contact stiffness is much less than that of the fingers, the palm response could be less than or comparable with that at the fingers, even if the effective mass of the palm would be greater than that of the fingers. However, because the effective mass, damping, and stiffness involved in each direction vary, the specific distribution and resonance frequency also varied with the direction.

\subsection{Vibration Power Transmission and Absorption}

The vibration power transmitted to each part of the hand is directly associated with the characteristics of the distributed impedances [17]. The identified distribution features indicate that the vibration power is primarily transmitted to the palm below a certain frequency in a power grip not only in the $z_{h}$-axis but also in the other two axes. At higher frequencies, the fingers could absorb more vibration power than the palm, especially in the finger resonance frequency range. If the power absorption is associated with the vibration-induced disorders, as it has been hypothesized [22-24], the potential for developing the finger disorders may be increased when the tool vibration is in the finger resonance frequency range. This further demonstrates the importance to examine the finger biodynamic responses.

The impedance phase measured at the fingers could be greater than $90^{\circ}$ at low frequencies, as seen in Figs. 4 and 5. This implies that the real part of the impedance and thereby the vibration power absorption would be negative. This further means that the VPA transmitted to the handle from the fingers is greater than that transmitted to fingers from the handle. This could be partially caused by the possible measurement uncertainty at the low frequencies; a relatively larger percent error at lower frequencies could occur because of the low force response due to low vibration magnitude of the excitation spectrum at such frequencies. However, the negative VPA could also be partially a natural phenomenon because the vibration power transmitted to the palm could also partially flow into the fingers and return to the handle. To verify this possibility qualitatively, a quick modeling analysis was performed by artificially increasing the palm contact stiffness and reducing the finger contact stiffness in the model from a reported study [30], which resulted in the predominant effect of the palm response. As expected, the negative finger VPA was replicated in the modeling. The model results also suggest that this phenomenon could occur in the $z_{h}$-axis in the presence of a sufficiently high palm force and/or sufficiently low finger force imparted to the handle. The large phase angle $\left(>90^{\circ}\right)$ could also partially result from the cross-axis interactions, similar to that observed in whole-body driving-point biodynamic responses to vibration [39]. These observations suggest that whereas the VPA of the entire hand-arm system can be estimated from the total hand impedance, it may not be appropriate to estimate the VPA absorbed in the fingers or palm directly from the impedance measured at the fingers or palm at low frequencies. While a model has been developed to estimate the VPA in the fingers in the $z_{h}$-axis [30], the 
impedance data measured in this study can be used to develop the models for estimating the finger VPAs in the other two directions.

This study also confirmed that the basic trends in the frequency dependency of the vibration power absorption for the entire hand-arm system are similar to that of the ISO frequency weighting (Fig. 9), not only in the $z_{h}$-axis but also in the other two directions. Consequently, the accelerations weighted with these four frequency dependency functions for the vast majority of tools are highly correlated to each other, as shown in Fig. 12. Such correlations mean that the total VPA in each direction is a vibration measure very similar to the current ISO frequency-weighted acceleration. This relationship also suggests that if the current ISO frequency-weighted acceleration could not provide a good prediction of the vibration-induced white finger, the total VPA method is unlikely to do a better job. However, because the weighting is derived primarily based on the subjective sensation data reported by Miwa [40,41], the total vibration power absorption is likely to be correlated with the subjective sensation or discomfort.

\subsection{Implications of the Results for Anti-vibration Glove Test and Evaluation}

The standardized anti-vibration glove evaluation defined in ISO 10819 (1996) [19] is based on the vibration transmissibility of a glove at the palm of the hand along the forearm direction or $z_{h}$-axis. Besides the dynamic properties of the glove, the measured transmissibility primarily depends on the biodynamic response at the palm. As demonstrated in a previous study [19], higher impedance around the first resonance generally corresponds to lower transmissibility of the glove in the middle and high frequencies. This mechanism suggests that the glove transmissibility measured in the $z_{h}$ direction with the standardized method generally overestimates its vibration reduction effectiveness for the following two reasons: (i) the $z_{h}$ direction impedance in the first resonance frequency range is the highest among those measured in the three directions; and (ii) the impedance at the palm is much higher than that at the fingers, particularly at lower frequencies. The mass effect of the palm adapter used in the standardized measurement could also further contribute to underestimation of the vibration transmissibility. Therefore, it is not appropriate to use the $z_{h}$ direction transfer function measured at the palm to estimate the actual vibration reduction effectiveness of anti-vibration gloves. The actual effectiveness can be more reliably examined using a tri-axial vibration test method at various locations on the hand-handle interface.

\subsection{Limitations of the study}

Many factors could affect the impedances distributed at the fingers and the palm of the hand. This study considered only one of many possible exposure conditions in the experiment. While the hand and arm postures and the applied hand forces used in this study may be considered representative to the average situations [4,21], the random excitation in each direction is only valid for determining the basic characteristics of the impedances. Different from the impedance under a single axis excitation, the impedance under the 3-D excitations may also vary with the magnitude ratios of the vibrations in the three directions and their coherences because of the possible cross-axis responses. Therefore, the 
impedances measured in this study may not be accurately representative of some working conditions. However, this should not affect the purposes of this study.

\section{Conclusion}

The distributed driving-point mechanical impedances vary greatly with the specific location of the hand, the vibration direction, and the individual. As a result, the two major resonances of the hand-arm system in the three directions are observed in a wide frequency range (20 to $300 \mathrm{~Hz}$ ). The impedance of the hand is primarily distributed at the palm below a certain frequency in the range of 50 to $100 \mathrm{~Hz}$, with the specific frequency transition depending on the vibration direction. However, the impedance at the fingers becomes comparable or higher than that at the palm at higher frequencies. While the impedance along the handle longitudinal axis is generally the smallest, the impedance along the forearm direction is generally the largest. The impedance in each direction in the plane vertical to the handle longitudinal axis is largely independent of vibration direction at high frequencies $(>250 \mathrm{~Hz})$, which suggests that only the hand tissues close the contact interface are involved in the response at such frequencies. These characteristics also suggest that the transmissibility of a glove measured with the standardized method is likely to overestimate the effectiveness of the glove for its vibration reduction. The results of this study also indicate that the vibration power absorption of the entire hand-arm system in each vibration direction or the sum of the VPAs in the three orthogonal directions is a vibration measure similar to the current ISO frequency-weighted acceleration.

\section{Disclaimers}

The content of this publication does not necessarily reflect the views or policies of the National Institute for Occupational Safety and Health (NIOSH), nor does mention of trade names, commercial products, or organizations imply endorsement by the U.S. Government.

\section{References}

[1] M.J. Griffin, Handbook of human vibration. London, Academic Press, 1990.

[2] R.G. Dong, J.Z. Wu, and D.E. Welcome, Recent advances in biodynamics of hand-arm system, Industrial Health 43(3) (2005) 449-71.

[3] M.J. Griffin, Foundations of hand-transmitted vibration standards, Nagoya Journal of Medical Science 57(supplement) (1994) 147-64.

[4] ISO-10068: Mechanical vibration and shock - Free, mechanical impedance of the human handarm system at the driving point. International Organization of Standard, Geneva, Switzerland, 1998.

[5] J.Z. Wu, K. Krajnak, D.E. Welcome, and R.G. Dong, Analysis of the dynamic strains in a fingertip exposed to vibration: correlation to the mechanical stimuli on mechanoreceptors, Journal of Biomechanics 39 (2006) 2445-2456. 
[6] J.Z. Wu, D.E. Welcome, R.G. Dong, Three-dimensional finite element simulations of the mechanical response of the fingertip to static and dynamic compressions, Computer Methods in Biomechanics and Biomedical Engineering 9(1) (2006) 55-63.

[7] J.Z. Wu, R.G. Dong, D.E. Welcome, and X.S. Xu, A method for analyzing vibration power absorption density in human fingertip, Journal of Sound and Vibration 329 (2010) 5600-5614.

[8] Jr. C.F Abrams, C.W. Suggs, Mechanical impedance simulation of vibrational characteristics of the hand-arm system. American Society of Agriculture Engineers 1970, Paper No. 70-523, 1-10.

[9] R. Gurram, S. Rakheja, and A.J. Brammer, Driving-point mechanical impedance of the human hand-arm system: Synthesis and model development, Journal of Sound and Vibration 180 (1995) 437-458.

[10] S. Rakheja, J.Z. Wu, R.G. Dong, and A.W. Schopper, A comparison of biodynamic models of the human hand-arm system for applications to hand-held power tools, Journal of Sound and Vibration 249(1) (2002), 55-82.

[11] R.G. Dong, D.E. Welcome, J.Z. Wu, and T.W. McDowell, Development of hand-arm system models for vibrating tool analysis and test rig construction, Noise Control Engineering Journal 56(1) (2008) 35-44.

[12] R.G. Dong, S. Rakheja, T.W. McDowell, D.E. Welcome, J.Z. Wu, Estimation of the biodynamic responses distributed at fingers and palm based on the total response of the hand-arm system, International journal of Industrial Ergonomics 40(4) (2010) 425-436.

[13] A.J. Besa, F.J. Valero, J.L. Suñer, J. Carballeira, Characterisation of the mechanical impedance of the human hand-arm system: The influence of vibration direction, hand-arm posture and muscle tension. International Journal of Industrial Ergonomics 37(3) 2007) 225-231

[14] S. Kihlberg, Biodynamic response of the hand-arm system to vibration from an impact hammer and a grinder, International Journal of Industrial Ergonomics 16 (1995) 1-8.

[15] J. Kinne, K. Latzel, T.H. Schenk, Application of two-hand impedance as basis for mechanical modeling, Proceedings of the $9^{\text {th }}$ International Conference on Hand-Arm Vibration, Nancy, France, 2001, 113-118.

[16] P. Marcotte, Y. Aldien, P.E. Boileau, S. Rakheja, J. Boutin, Effect of handle size and handhandle contact force on the biodynamic response of the hand-arm system under zh-axis vibration, Journal of Sound and Vibration 283(3-5) (2005) 1071-1091.

[17] R.G. Dong, D.E. Welcome, T.W. McDowell, J.Z. Wu, A.W. Schopper, Frequency weighting derived from power absorption of fingers-hand-arm system under $\mathrm{Z}_{\mathrm{h}}$-axis, Journal of Biomechanics 39 (2006) 2311-2324.

[18] R.A. Toupin, Saint-Venant's principle. Archive for Rational Mechanics and Analysis 18 (1965) 83-96.

[19] R.G. Dong, T.W. McDowell, D.E. Welcome, C. Warren, J.Z. Wu, and S. Rakheja, Analysis of anti-vibration gloves mechanism and evaluation methods, Journal of Sound and Vibration 321(2009) 435-453 . 
[20] ISO 13753: Mechanical vibration and shock -- hand-arm vibration -- method for measuring the vibration transmissibility of resilient materials when loaded by the hand-arm system. International Organization for Standardization, Geneva, Switzerland, 1999.

[21] ISO 10819: Mechanical vibration and shock -- hand-arm vibration -- method for the measurement and evaluation of the vibration transmissibility of gloves at the palm of the hand. International Organization for Standardization, Geneva, Switzerland, 1996.

[22] F. Pradko, R.A. Lee, and J.D. Greene, Human vibration-response theory, American Society of Mechanical Engineers (1965) Paper No. 65-WA/HUF-19.

[23] I.M. Lidström, Vibration injury in rock drillers, chiselers, and grinders. Some views on the relationship between the quantity of energy absorbed and the risk of occurrence of vibration injury, Proceedings of the International Conference on Hand-Arm Vibration, Cincinnati, $\mathrm{OH}$, USA, 1977, 77-83.

[24] J.S. Cundiff, Energy dissipation in human hand-arm exposed to random vibration, Journal of the Acoustical Society of America 59 (1976) 212-214.

[25] R.G. Dong, A.W. Schopper, T.W. McDowell, D.E. Welcome, J.Z. Wu, W.P. Smutz, C. Warren, S. Rakheja, Vibration energy absorption (VEA) in human fingers-hand-arm system, Medical Engineering \& Physics 26(7) (2004) 483-492.

[26] L. Burström and R. Lundström, Absorption of vibration energy in the human hand and arm, Ergonomics 37 (1994) 879-890.

[27] ISO 5349-1: Mechanical vibration - Measurement and evaluation of human exposure to handtransmitted vibration - Part 1: General requirements. International Organization for Standardization, Geneva, Switzerland, 2001.

[28] R.G. Dong, J.Z. Wu, T.W. McDowell, D.E. Welcome, A.W. Schopper, Distribution of mechanical impedance at the fingers and the palm of human hand, Journal of Biomechanics 38(5) (2005) 1165-1175.

[29] R.G. Dong, J.H. Dong, J.Z. Wu, S. Rakheja, Modeling of biodynamic responses distributed at the fingers and the palm of the human hand-arm system, Journal of Biomechanics 40 (2007) 2335-40.

[30] J. H. Dong, R.G. Dong, S. Rakheja, D.E. Welcome, T.W. McDowell, and J.Z. Wu, A method for analyzing absorbed power distribution in the hand and arm substructures when operating vibrating tools, Journal of Sound and Vibration 311 (2008) 1286-1309.

[31] S. Rakheja, R.G. Dong, D.E. Welcome, A.K.W. Ahmed, A preliminary study of cross-axis coupling effects in biodynamic response of the hand-arm system, Proceedings of the $11^{\text {th }}$ International Conference on Hand-Arm Vibration, Bologna, Italy, 2007.

[32] R.G. Dong, D.E. Welcome, T.W. McDowell, X.S. Xu, J.Z. Wu, and S. Rakheja, Mechanical Impedances Distributed at the Fingers and Palm of the Human Hand Subjected to 3-D Vibrations. Proceedings of the $3^{\text {rd }}$ American Conference on Human Vibration (2010), Iowa City, USA. 
[33] R.G. Dong, D.E. Welcome, R. McCormick, A novel 3-D hand-arm vibration test system and its preliminary evaluation. Proceedings of the $1^{\text {st }}$ American Conference on Human Vibration (2006), Morgantown, WV, USA.

[34] M.J. Griffin, Measurement, evaluation, and assessment of occupational exposures to handtransmitted vibration, Occupational Environmental Medicine 54(2) (1997) 73-89.

[35] R.G. Dong, D.E. Welcome, T.W. McDowell, and J.Z. Wu. Analysis of handle dynamics-induced errors in hand biodynamic measurements. Journal of Sound and Vibration 318(4-5) (2008) 13131333.

[36] I. Pyykkö, M. Färkkilä, J. Toivanen, O. Korhonen, and J. Hyvärinen, Transmission of vibration in the hand-arm system with special reference to changes in compression force and acceleration, Scandinavian Journal of Work, Environment \& Health 2 (1976) 87-95.

[37] D. Reynolds, and E.N. Angevine, Hand-arm vibration, part II: Vibration transmission characteristics of the hand and arm, Journal of Sound and Vibration 51 (1977) 255-265.

[38] A. Sörensson, and R. Lundström, Transmission of vibration to the hand, Journal of Low Frequency Noise and Vibration 11 (1992) 14-22.

[39] N. Nawayseh and M.J. Griffin, A model of the vertical apparent mass and the fore-and-aft crossaxis apparent mass of the human body during vertical whole-body vibration, Journal of Sound and Vibration 319(1-2) (2009) 719-730.

[40] T. Miwa, Evaluation Methods for Vibration Effect: Part 6. Measurements of Unpleasant and Tolerance Limit Levels for Sinusoidal Vibrations. Industrial Health 6 (1968) 18-27.

[41] A.J. Brammer, Dose-response relationship for hand-transmitted vibration. Scandinavian Journal of Work Environment and Health 12 (1986) 284-288. 


\section{List of Tables and Figures}

Table 1: Subject anthropometry (hand length $=$ tip of middle finger to crease at wrist; hand breadth = the width measured at metacarpal.)

Figure 1: Subject posture and measurement set-up that includes a closed-loop controlled vibration supply system, a vibration and response measurement system, a grip force measurement and display system, and a push force measurement and display system.

Figure 2: A pictorial view of the 3-D hand-arm test system, together with a test subject (Note: The directions of the controlled excitations do not depend on the orientations of the shakers but depend on the orientation of the tri-axial accelerometer fixed in the instrumented handle. The accelerometer was arranged according to the directions shown in the figure).

Figure 3: A sketch of the instrumented handle used for the measurement of the finger and palm mechanical impedances.

Figure 4: The mechanical impedances distributed at the fingers and palm of the hand-arm system in the $x_{h}$ direction: (a) fingers; (b) palm; (c) hand. ( Subject 1; $\square$ Subject 2; + Subject 3; $\triangle$ Subject 4; - Subject 5; $\times$ Subject 6; $\square$ Subject 7).

Figure 5: The mechanical impedances distributed at the fingers and palm of the hand-arm system in the $y_{h}$ direction: (a) fingers; (b) palm; (c) hand. ( Subject 1; $\square$ Subject 2; + Subject 3; \ Subject 4; $\bullet$ Subject 5; $\times$ Subject 6; $\square$ Subject 7).

Figure 6: The mechanical impedances distributed at the fingers and palm of the hand-arm system in the $z_{h}$ direction: (a) fingers; (b) palm; (c) hand. ( Subject 1; - Subject 2; + Subject 3; $\triangle$ Subject 4; $\bullet$ Subject 5; $\times$ Subject 6; $\square$ Subject 7).

Figure 7: The distribution of the mechanical impedances in the three orthogonal directions: (a) $x_{h^{-}}$ axis; (b) $y_{h}$-axis; (c) $z_{h}$-axis. ( fingers; $\bullet$ palm; $\bullet$ hand).

Figure 8: The effects of the vibration direction on the mechanical impedances: (a) fingers; (b) palm; (c) hand. ( $x_{h}$-axis; $\square y_{h}$-axis; $\bullet z_{h}$-axis).

Figure 9: Comparisons of the ISO frequency weighting (—_) defined in ISO 5349-1 (2001) [27] with the frequency dependencies of the vibration power absorptions of the entire hand-arm system in the three directions ( $\bullet x_{h}$ direction; $y_{h}$ direction; $\bullet z_{h}$ direction), which are derived from Eq.(4) using the real parts of the mechanical impedances in the three 
directions. The reference frequency is $20 \mathrm{~Hz}$ in the normalization of the VPA frequency dependencies.

Figure 10: Comparisons of the impedances distributed at fingers and the palm of the hand with those reported before: (a) fingers; (b) palm. (• measured under $30 \mathrm{~N}$ grip and $50 \mathrm{~N}$ push in the current study; - measured with eight subjects under $30 \mathrm{~N}$ grip and $45 \mathrm{~N}$ push reported in a previous study [17]).

Figure 11: Comparisons of the mean impedances of the entire hand-arm system with the data recommended in ISO 10068 (1998) [4]: (a) $x_{h}$ direction; (b) $y_{h}$ direction; (c) $z_{h}$ direction (— upper or lower limit in ISO 10068; ...... mean values in ISO 10068; • current values in $x_{h}$; current values in $y_{h} ; \bullet$ current values in $z_{h}$ ).

Figure 12: Correlations between the ISO frequency-weighted accelerations and the VPA-weighted accelerations (the accelerations weighted using the frequency dependency of the VPA in each direction), which are calculated from the vibration spectra of 20 different tools reported by Griffin [34]: (a) $x_{h}$ direction; (b) $y_{h}$ direction; (c) $z_{h}$ direction ( $\bullet$ values in $x_{h}$; v values in $y_{h} ; \quad$ values in $z_{h}$; trendline). 
Table 1

\begin{tabular}{ccccc} 
Subject & $\begin{array}{c}\text { Height } \\
(\mathbf{c m})\end{array}$ & $\begin{array}{c}\text { Weight } \\
(\mathbf{k g})\end{array}$ & $\begin{array}{c}\text { Hand } \\
\text { Length } \\
(\mathbf{m m})\end{array}$ & $\begin{array}{c}\text { Hand } \\
\text { Breadth } \\
(\mathbf{m m})\end{array}$ \\
\hline 1 & 181.6 & 99.79 & 200 & 94 \\
2 & 185.4 & 69.10 & 192 & 86 \\
3 & 182.9 & 68.95 & 192 & 84 \\
4 & 176.5 & 79.83 & 193 & 83 \\
5 & 180.3 & 88.45 & 192 & 89 \\
6 & 180.8 & 80.70 & 200 & 90 \\
7 & 179.1 & 87.00 & 190 & 89 \\
\hline Mean & 180.9 & 81.97 & 194 & 88 \\
SD & 2.8 & 11.00 & 4 & 4 \\
\hline
\end{tabular}


Figure 1

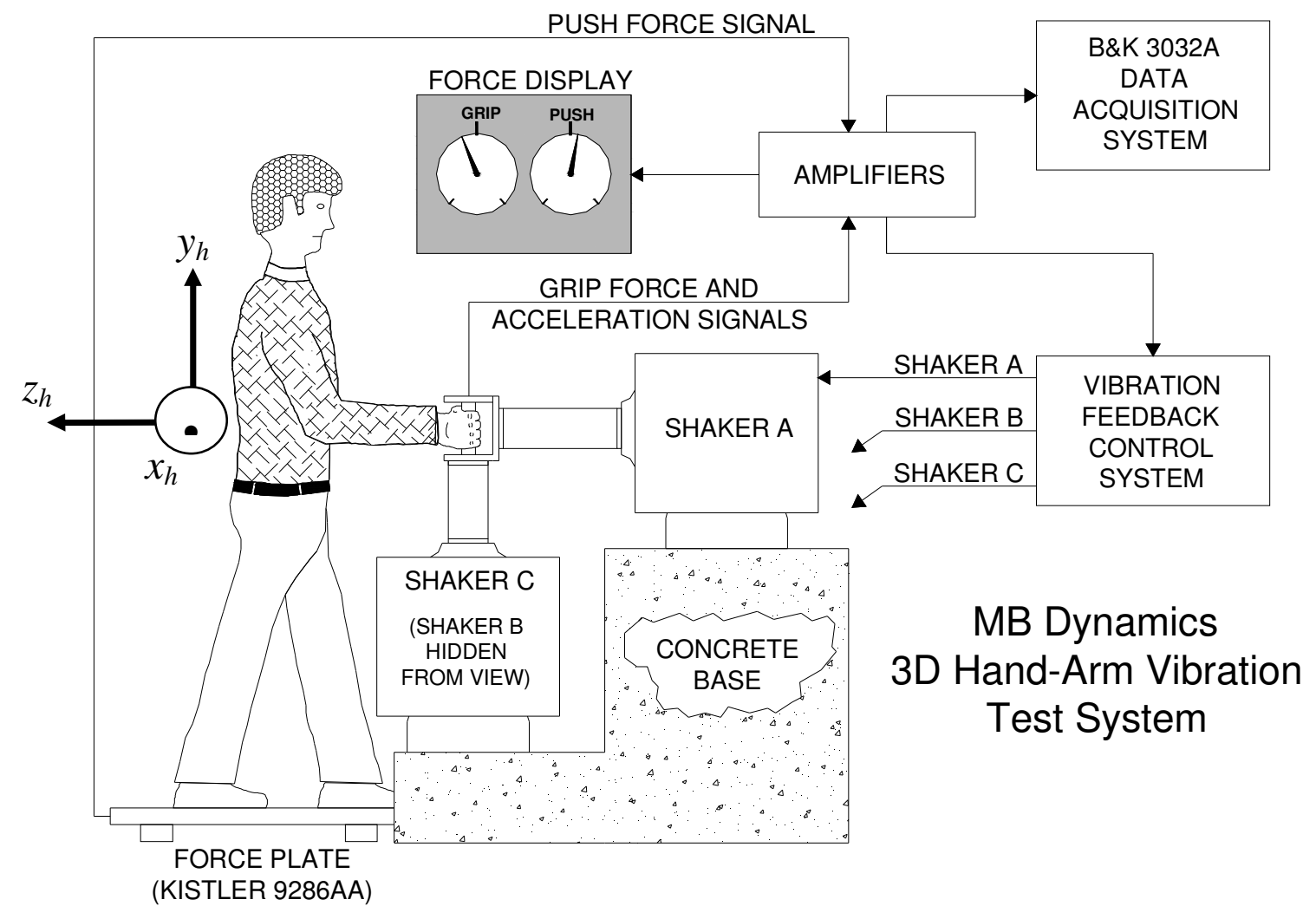


Figure 2

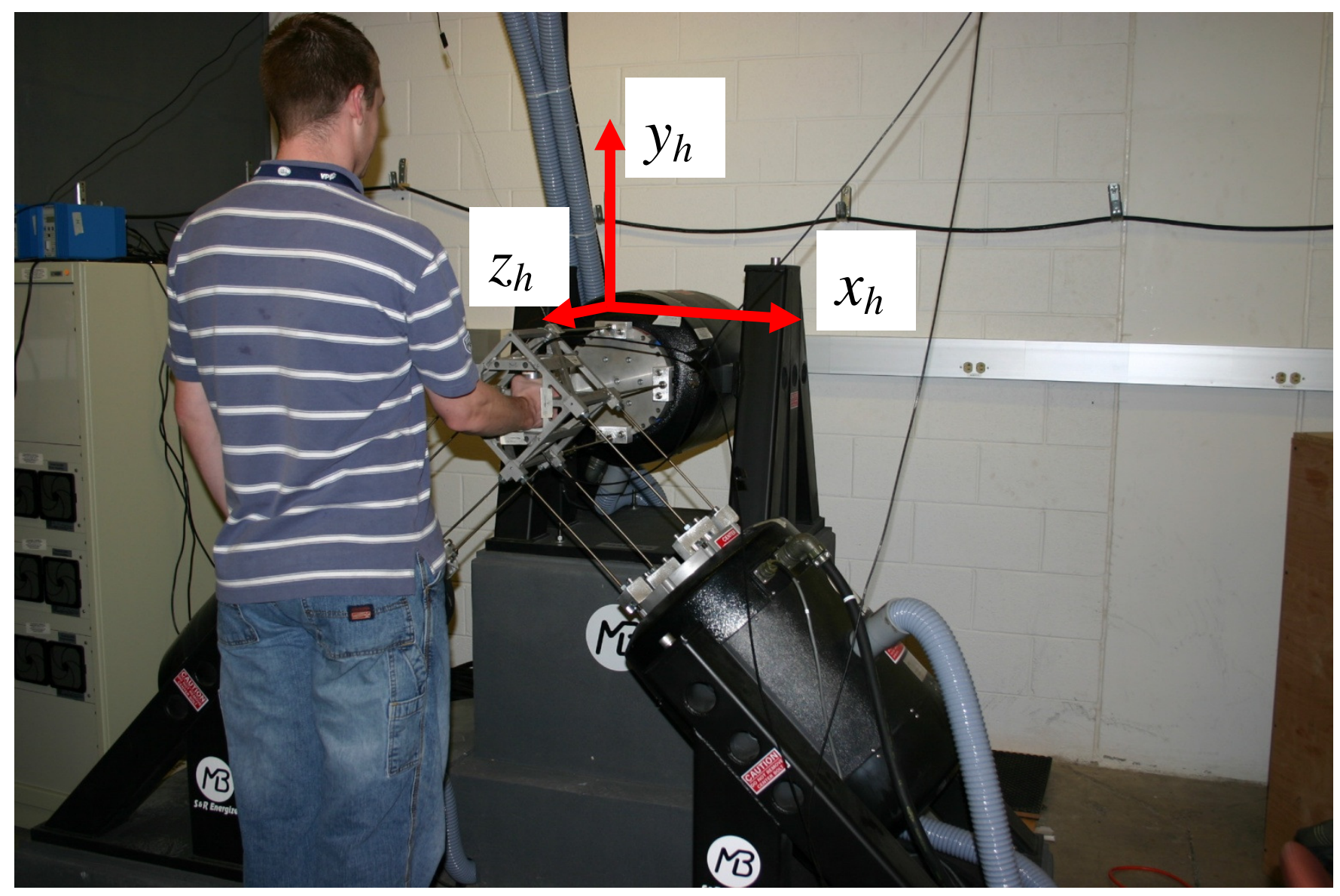


Figure 3

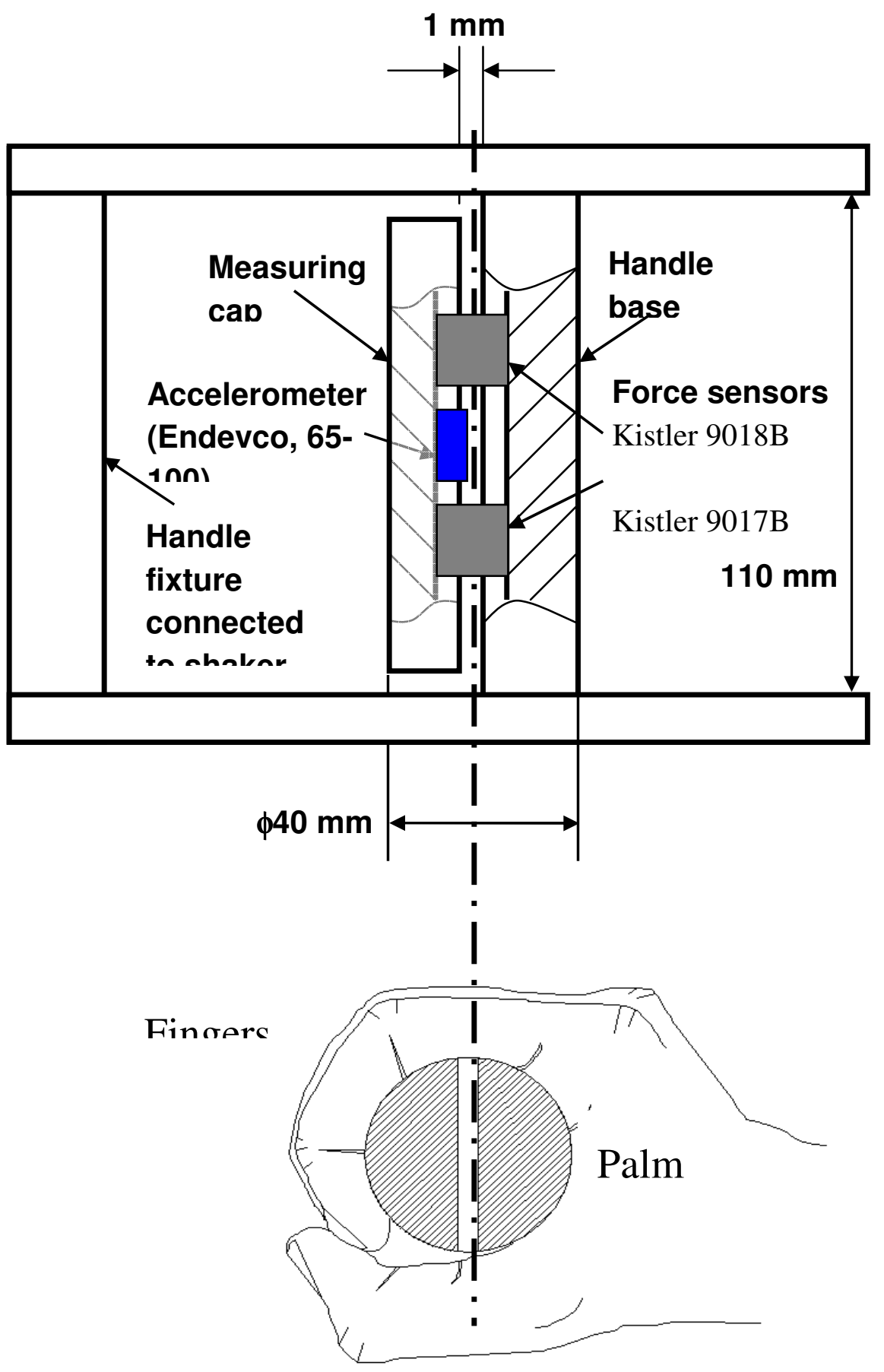


Figure 4
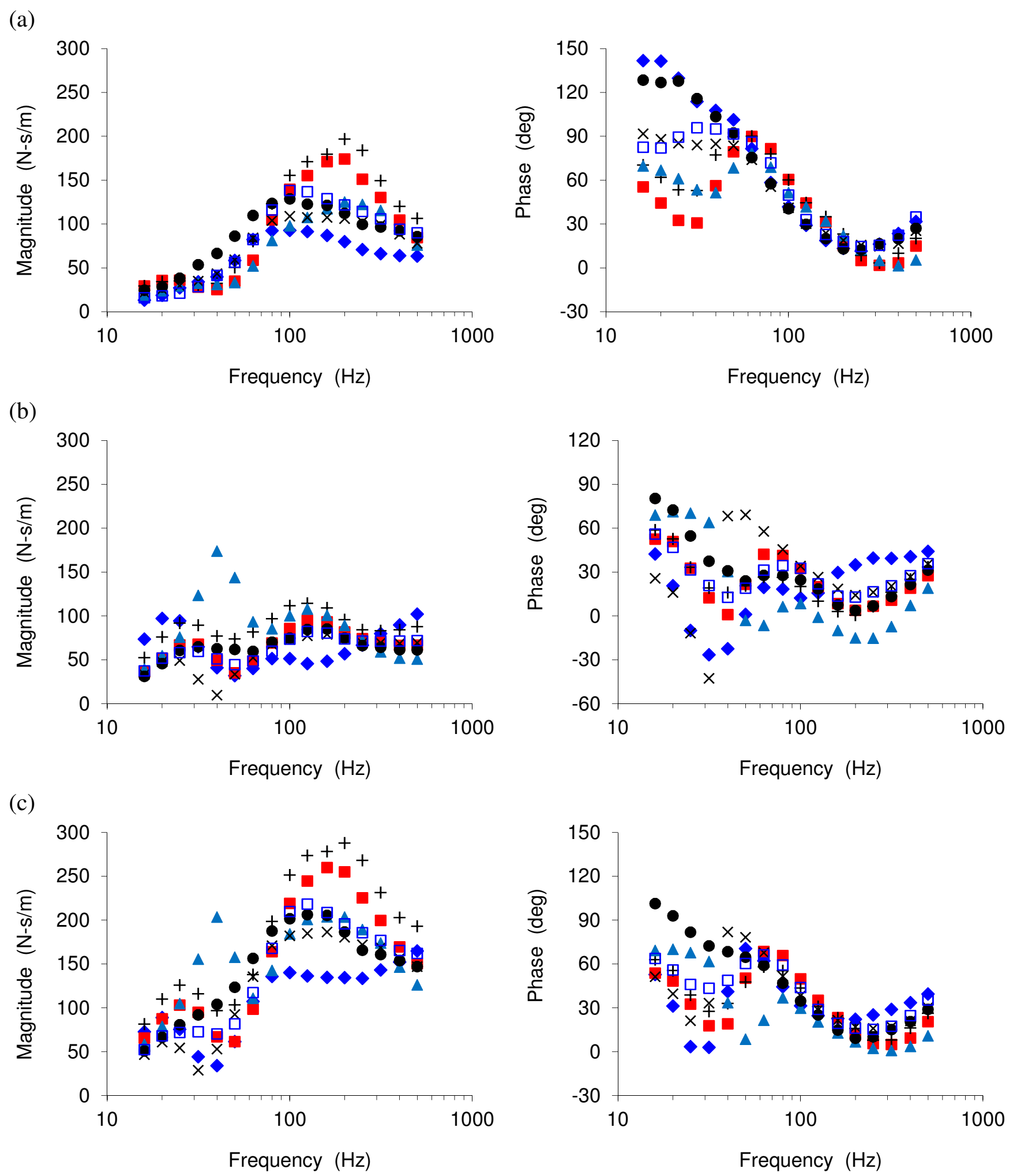
Figure 5
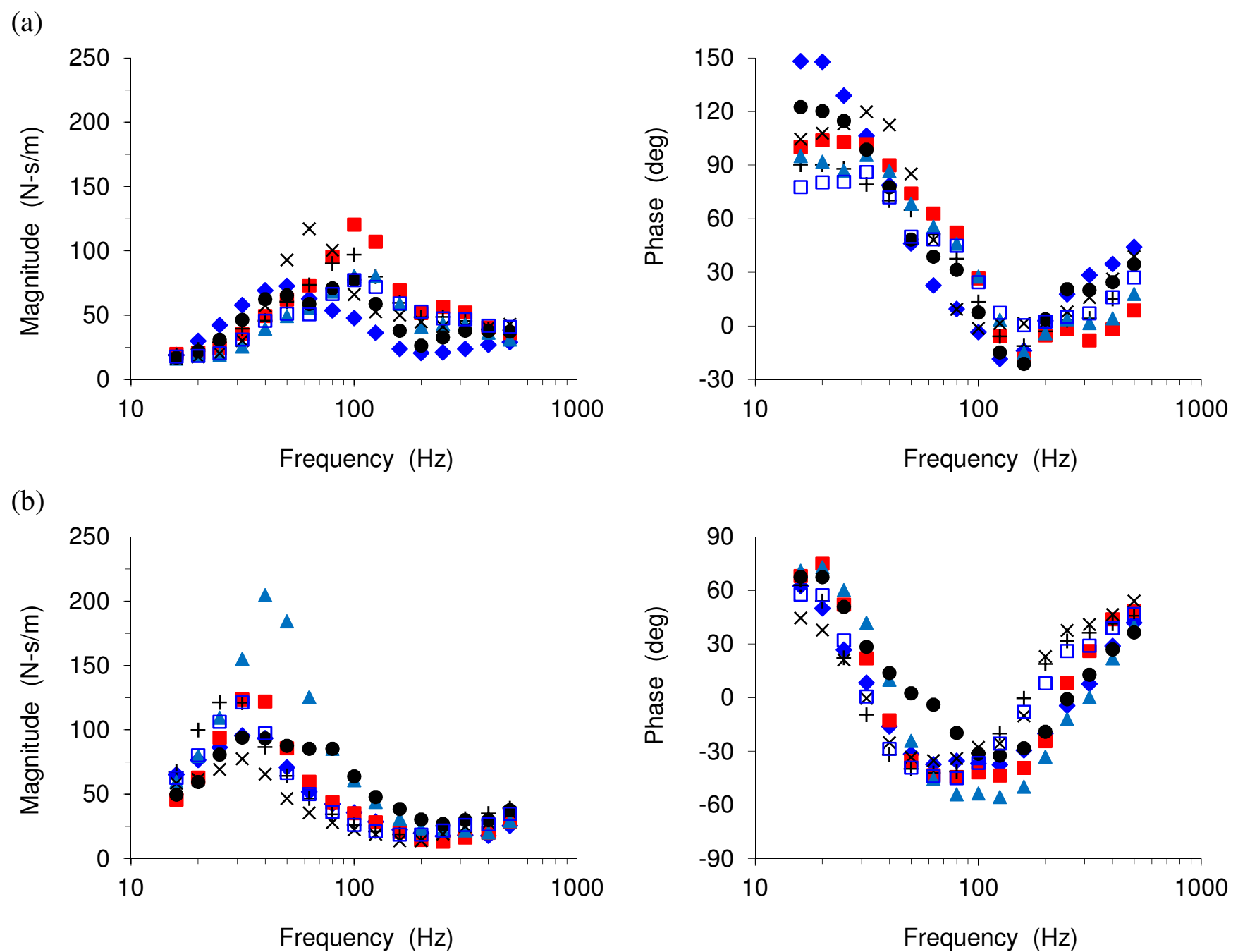

(c)
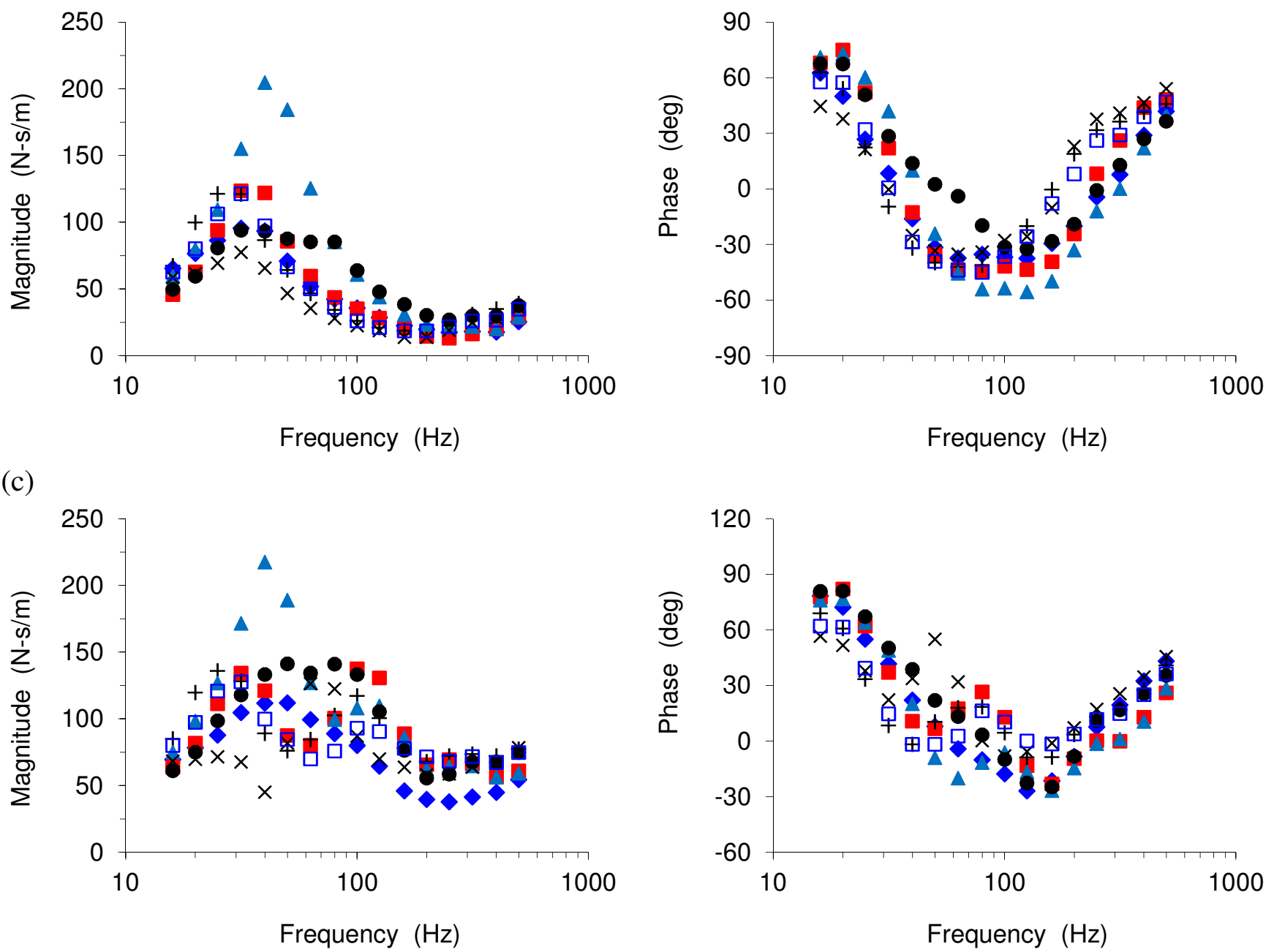
Figure 6
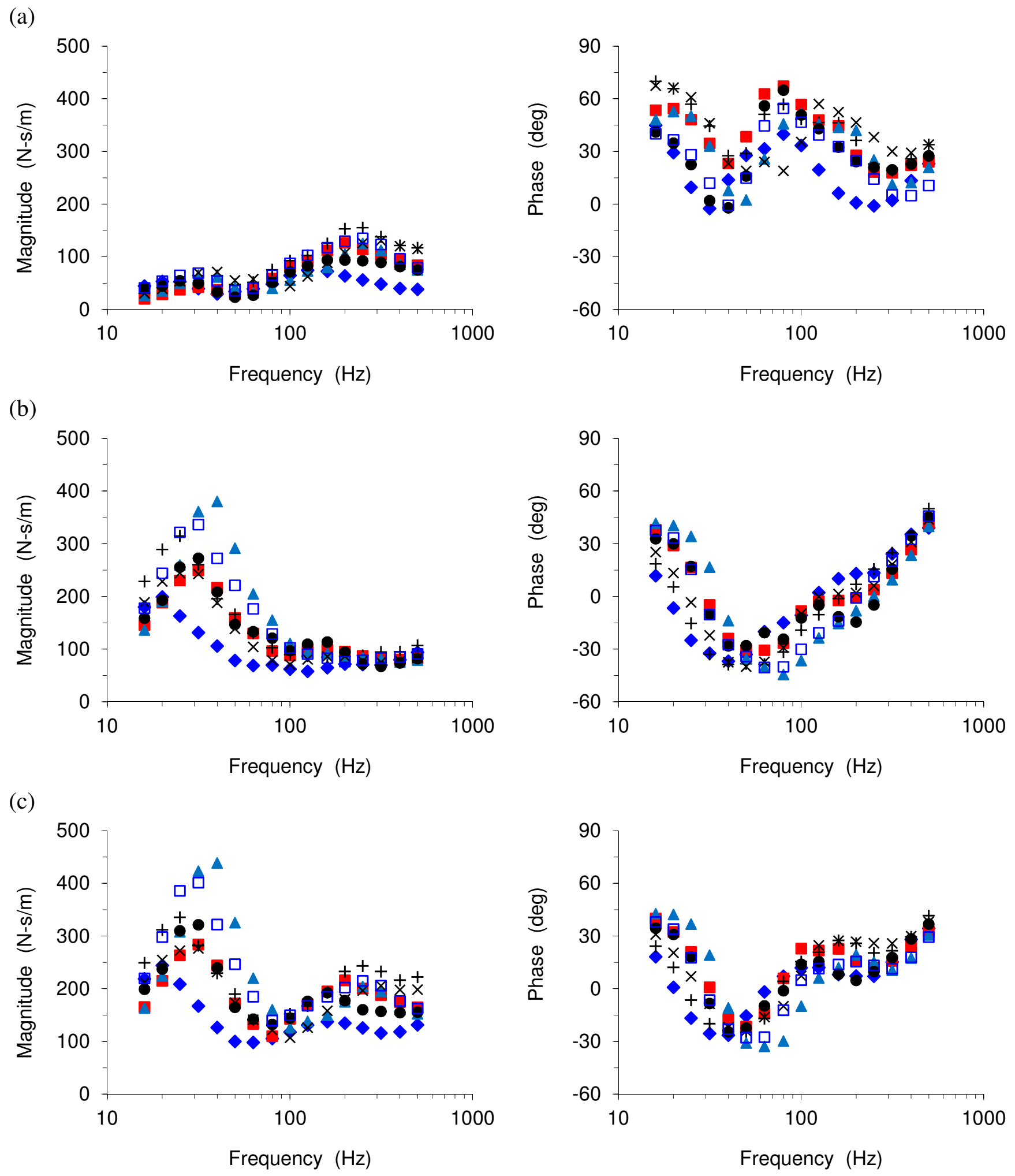
Figure 7

(a)
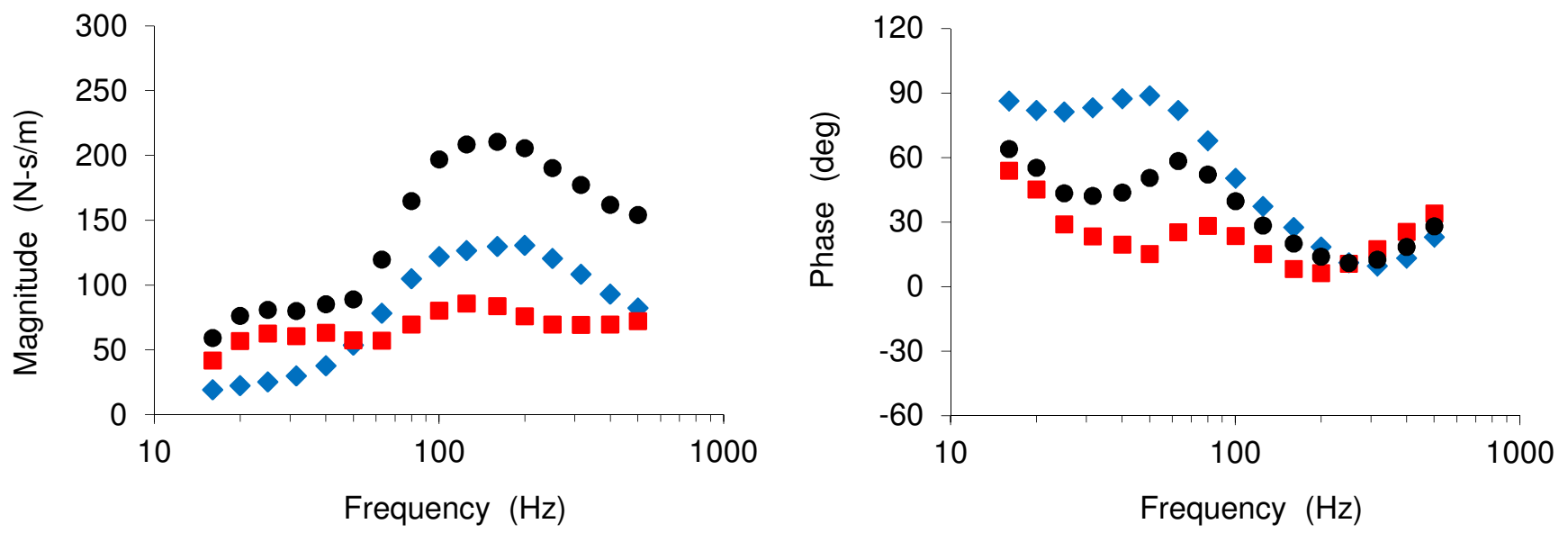

(b)

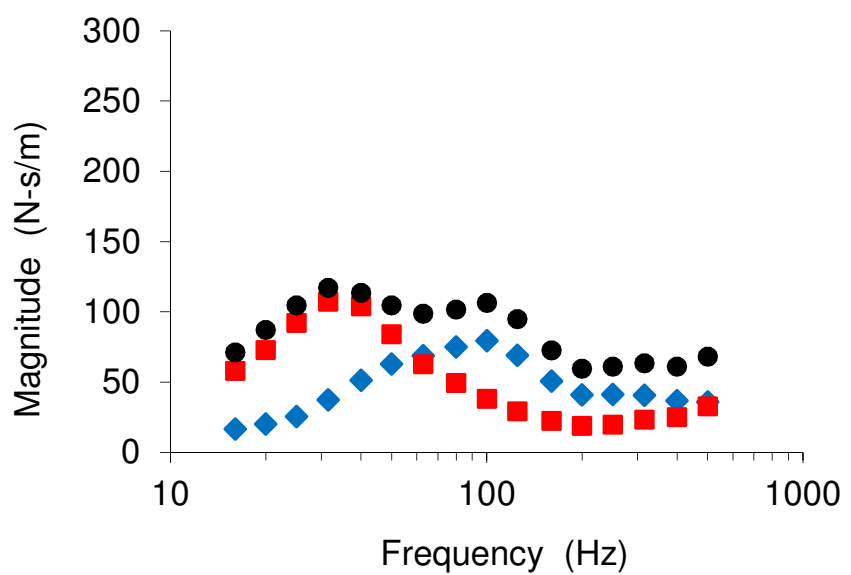

(c)
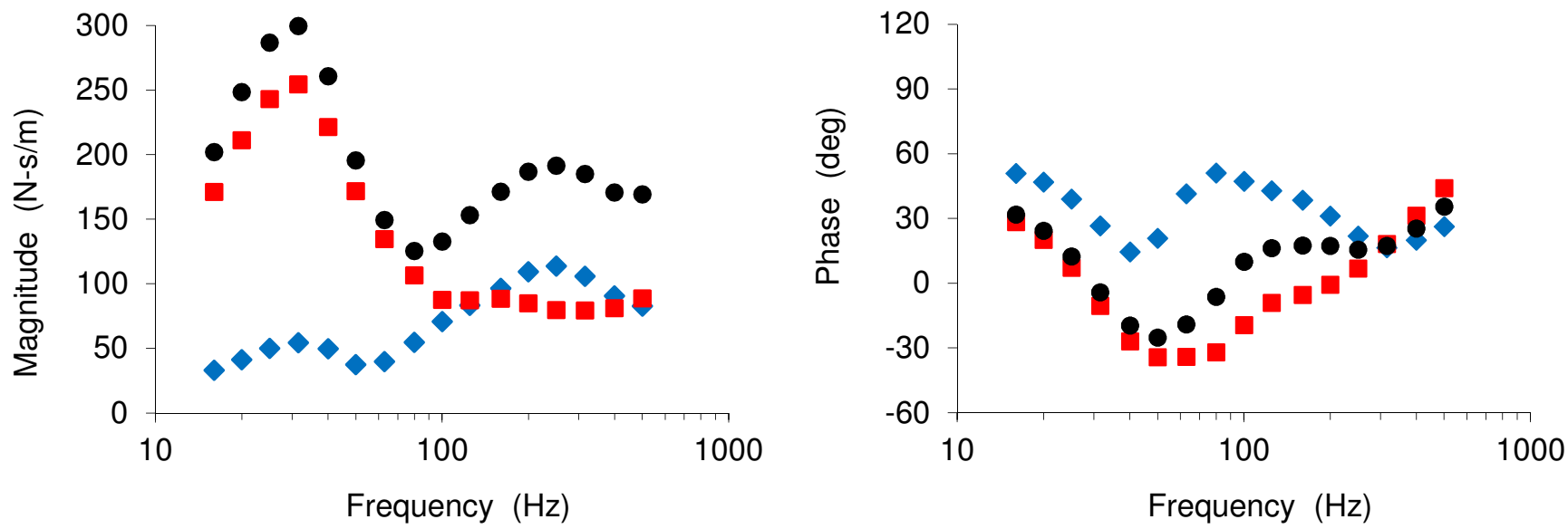
Figure 8
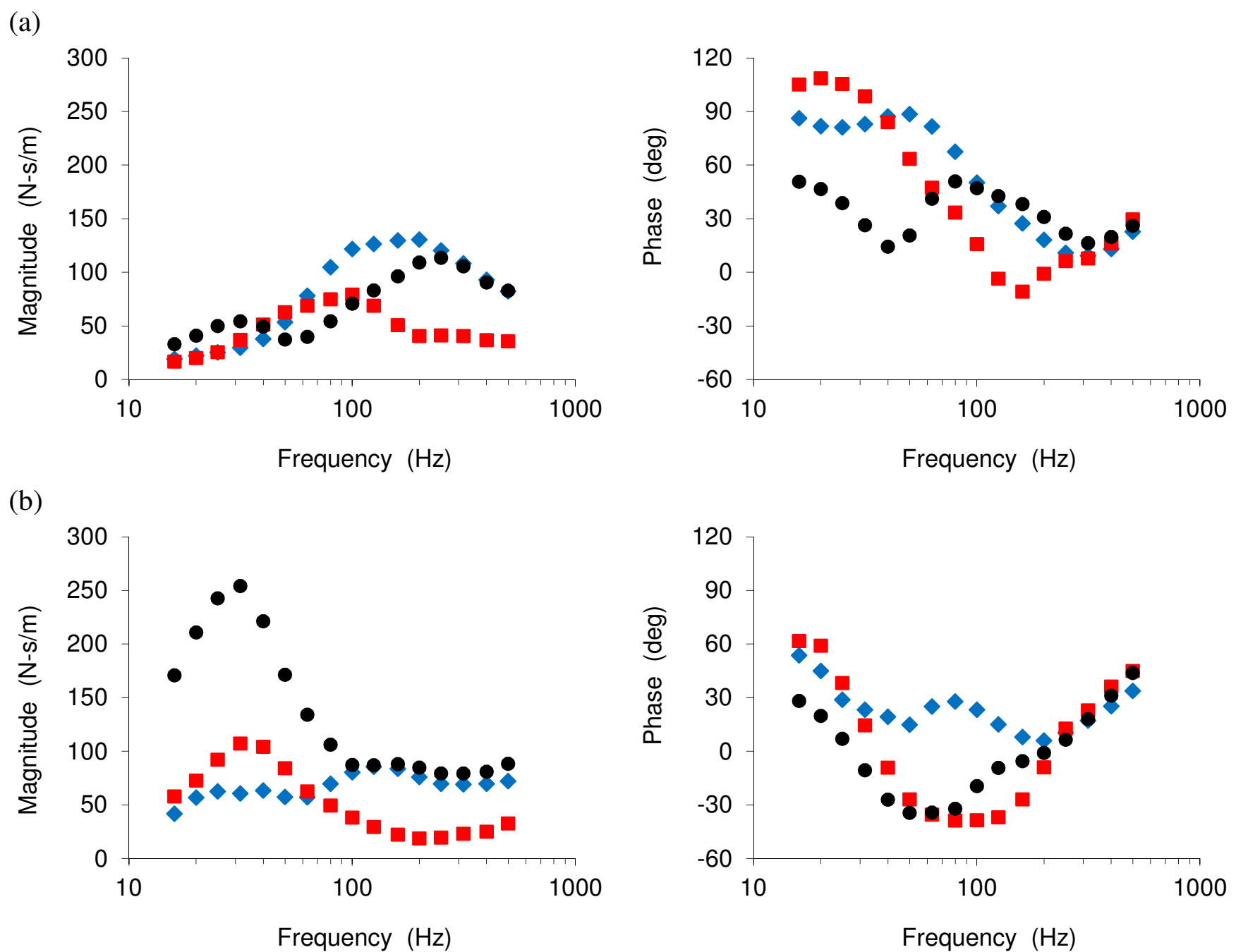

(c)
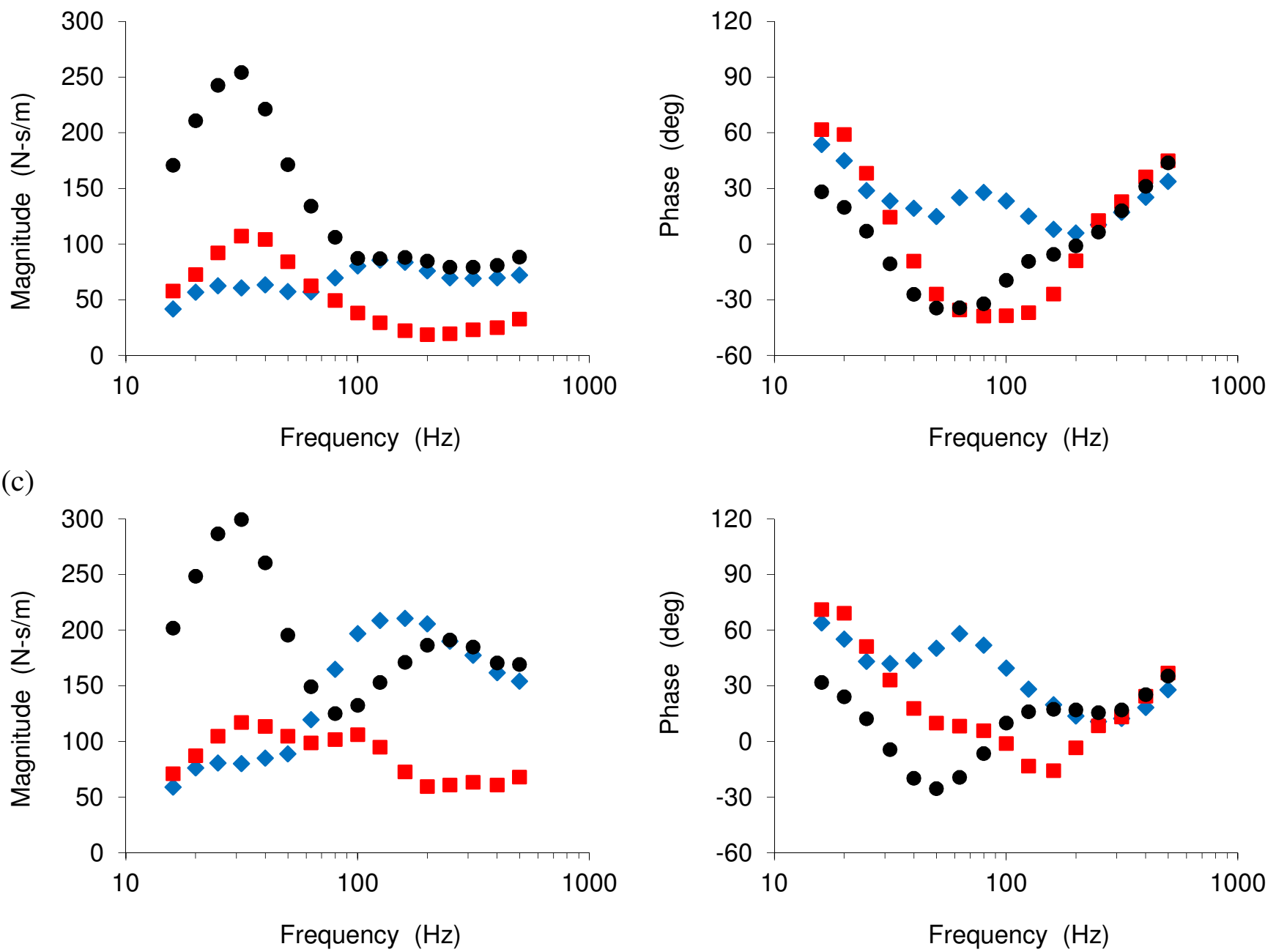
Figure 9

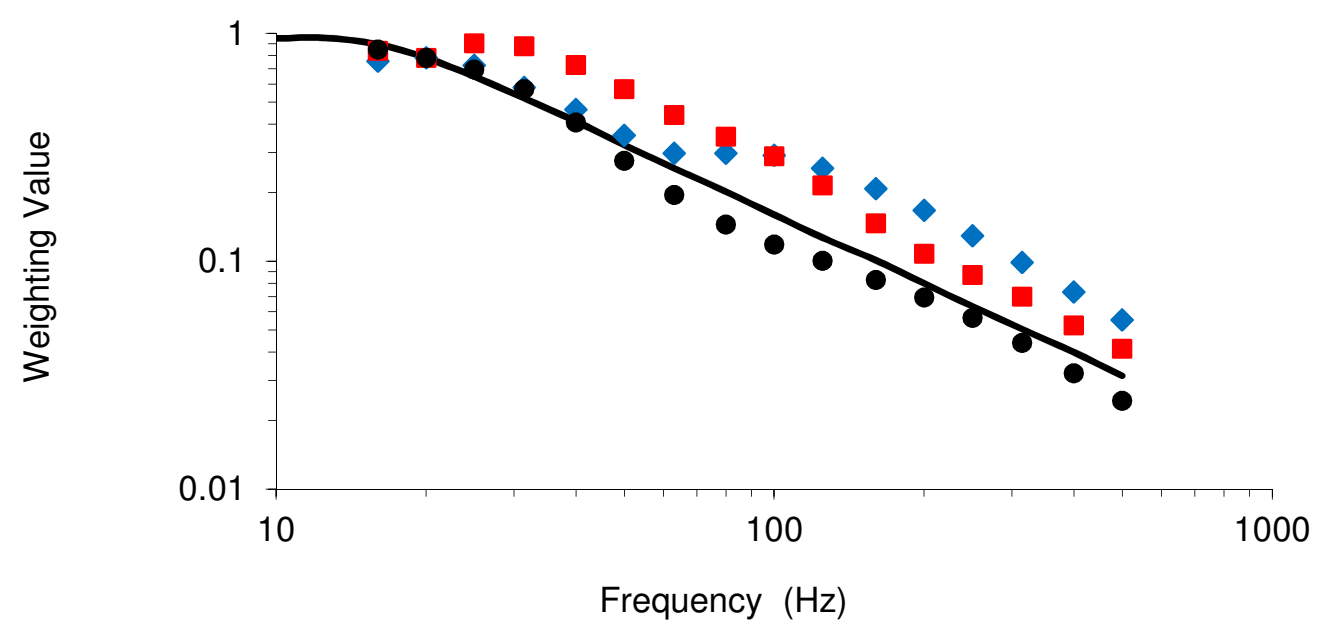

Figure 10

(a)
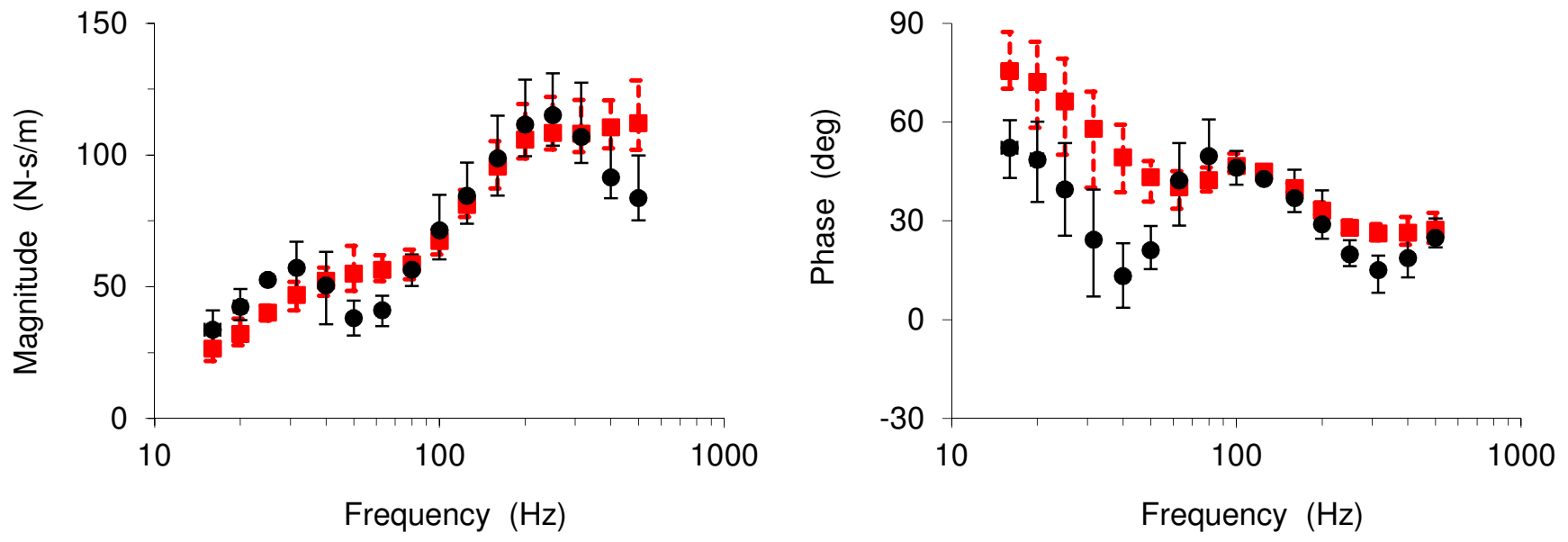

(b)
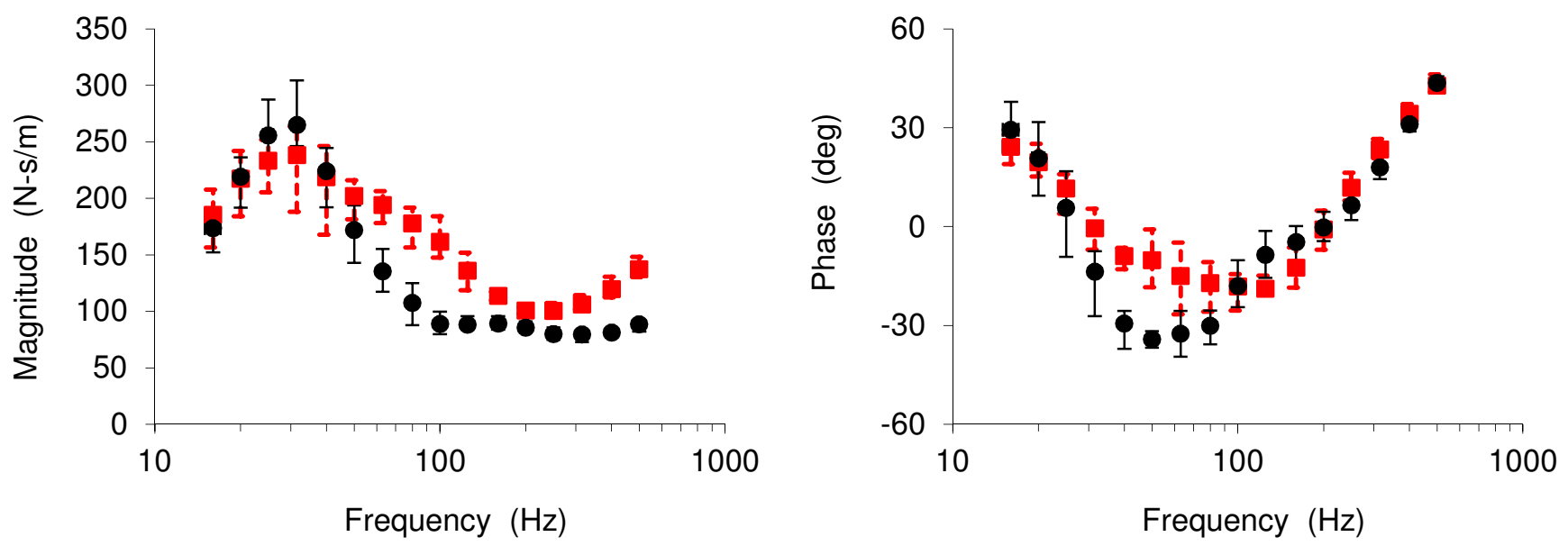
Figure 11

(a)

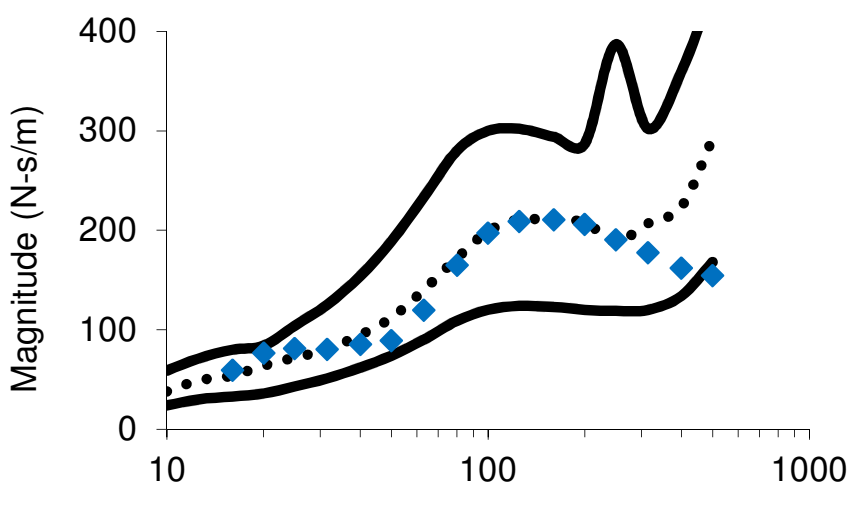

Frequency $(\mathrm{Hz})$

(b)

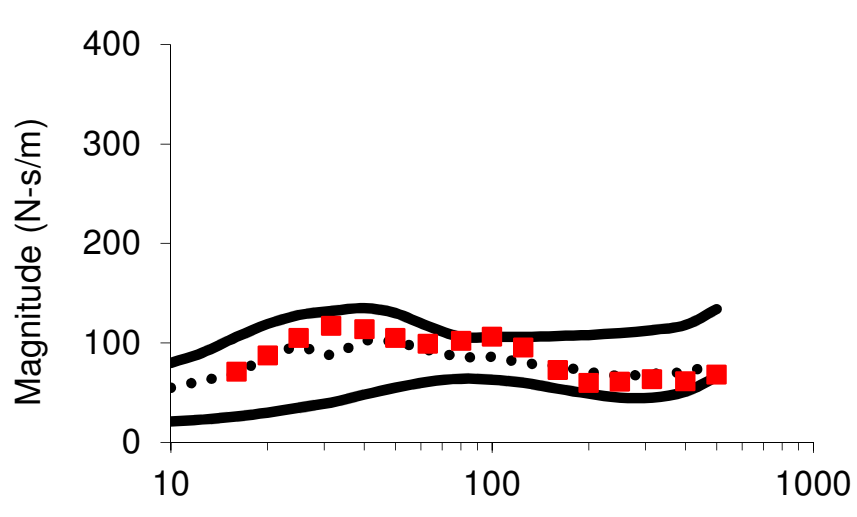

Frequency $(\mathrm{Hz})$

(c)

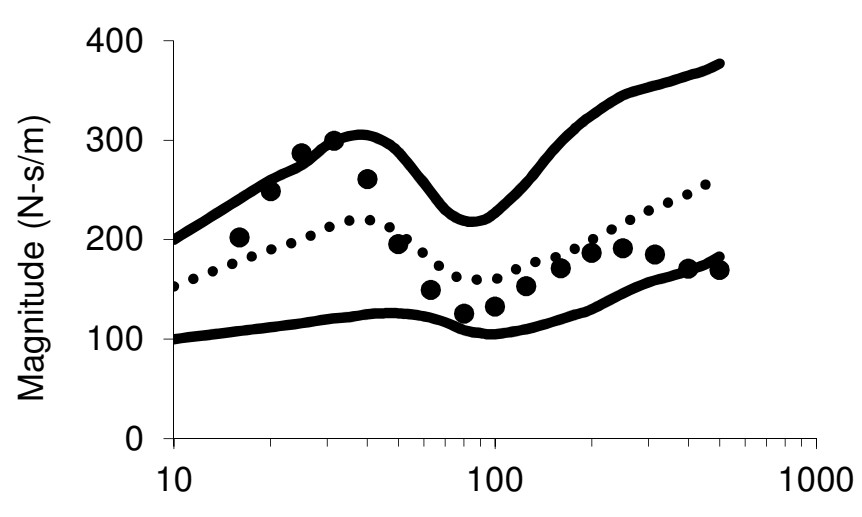

Frequency $(\mathrm{Hz})$
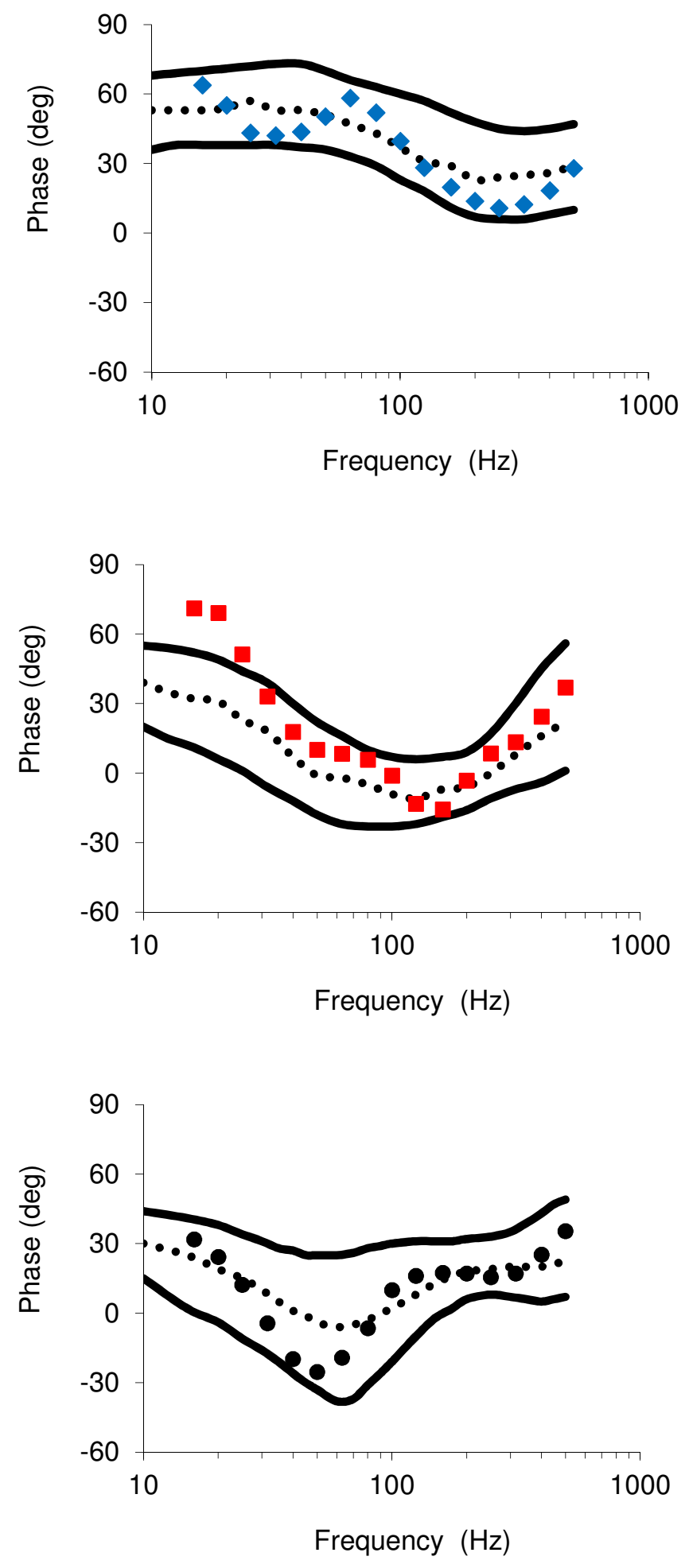
Figure 12

(a)

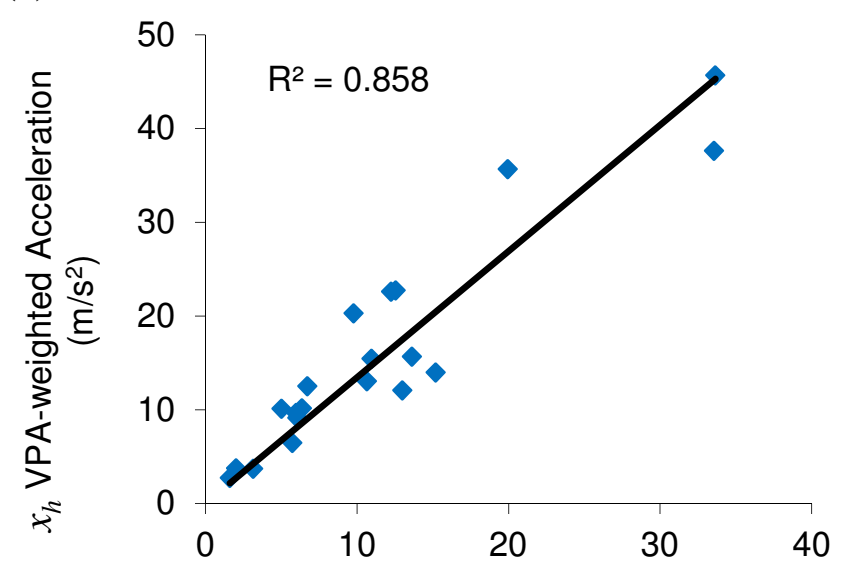

ISO Weighted Acceleration $\left(\mathrm{m} / \mathrm{s}^{2}\right)$ (b)

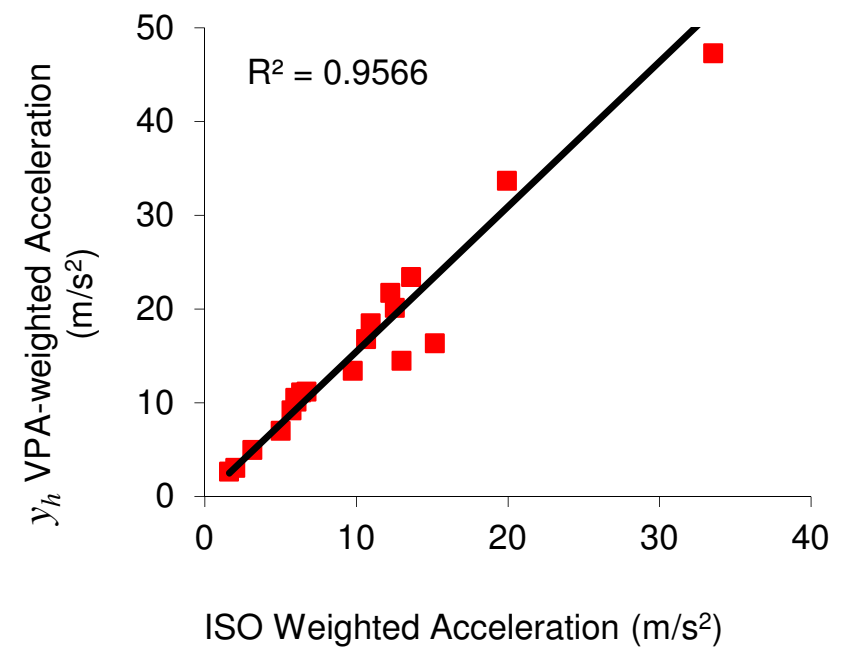

(c)

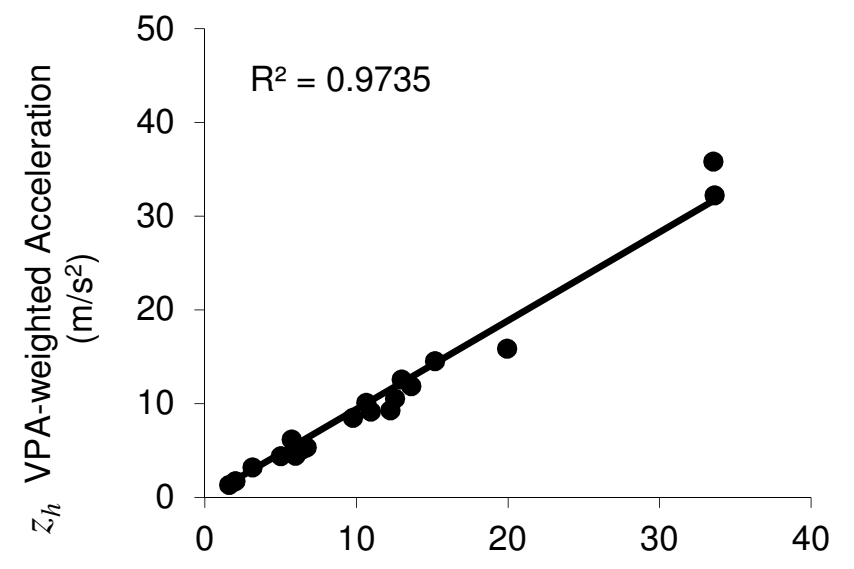

ISO Weighted Acceleration $\left(\mathrm{m} / \mathrm{s}^{2}\right)$ 